\title{
Estudo de modelos para epidemias no curso de Cálculo Numérico
}

\author{
Júlia Beatriz Meira (i) Bruno Telch dos Santos (i)
}

\begin{abstract}
Resumo
Apresentamos em uma linguagem bastante simples os resultados de uma experiência professoraluno para a avaliação do curso de Cálculo Numérico. Seguindo a metodologia abordada em [8], o texto apresenta uma atividade do curso de Cálculo Numérico, que tem um problema de ecologia matemática como eixo norteador - a saber, envolve o tema de epidemias - e busca fazer um entendimento geral dessa dinâmica, combinando a teoria elementar com ferramentas básicas do curso de Cálculo numérico, e finalmente comparando com dados reais da epidemia na China. O texto foi construído com o objetivo de introduzir modelos de epidemias com uma linguagem simples, sendo as abordagens numéricas meramente ilustrativas.
\end{abstract}

Palavras-chave: Cálculo Numérico, Modelos epidemiológicos, Matlab, Ecologia Matemática.

\begin{abstract}
We present in a very simple language the results of a teacher-student experience for the evaluation of the Numerical Calculus course. Following the methodology covered in [8], the text presents an activity of the Numerical Calculus course, which has a mathematical ecology problem as a guiding axis, namely, it involves the theme of epidemics, and seeks to make a general understanding of this dynamic by combining elementary theory with basic tools of the Numerical Calculus course, and finally comparing with actual data from the epidemic in China. The text was constructed with the objective of introducing epidemic models in a simple language and the numerical approaches are merely illustrative.
\end{abstract}

Keywords: Numerical Calculus, Epidemiological models, MATLAB, Mathematical Ecology.

\section{Introdução}

No dia 11 de Março de 2020 a Organização Mundial da Saúde (OMS) elevou o estado da contaminação de Covid-19, doença causada pelo novo coronavírus (Sars-Cov-2), ao status de pandemia. A epidemia começou com casos relacionados a infecções respiratórias em Wuhan, cidade chinesa, no início de dezembro de 2019. Tal doença desconhecida tornou-se alarmante no país com o descontrole da expansão do vírus ao acarretar inúmeros contaminados. Após a confirmação da existência do vírus em países vizinhos, reconheceram-se o surto do novo coronavírus e a gravidade da situação os quais implicaram medidas de controle da expansão da moléstia na China, como a quarentena. Todavia, salienta-se o contato dos chineses em outros países, o qual acarretou a propagação da 
doença em outros continentes. Isto posto, o novo vírus estabeleceu-se como emergência de saúde pública de interesse internacional pela OMS. Diante disso, medidas de restrição implementaram-se nos países a fim de conter a expansão do coronavírus, entretanto, a quantidade de doentes supria a capacidade dos hospitais em vários países e o número de mortes elevava-se. Por conseguinte, em virtude do aumento de casos de Covid-19, de países contaminados e de mortes, a doença foi reconhecida como uma pandemia.

Grupos de saúde pública como o Centro para Controle e Prevenção de Doenças (CDC-EUA) e a Organização Mundial da Saúde (OMS) vêm monitorando a pandemia e publicando atualizações em seus sites, onde foram emitidas recomendações para prevenir e tratar a doença. Os sinais e sintomas do Covid-19 costumam aparecer entre o segundo e o décimo quarto dia após a exposição. Sinais e sintomas mais comuns podem incluir febre, tosse e cansaço. Outros sintomas que podem ser destacados são a falta de ar ou dificuldade em respirar, dores musculares, arrepios, dor de garganta, perda de paladar ou olfato, dores de cabeça e dores no peito. Sintomas menos comuns que também foram relatados incluem náusea, vômito e diarreia. As crianças apresentam sintomas semelhantes aos adultos e geralmente apresentam doenças leves. A gravidade dos sintomas varia de muito leve a grave, algumas pessoas podendo ter apenas alguns desses sintomas e outras podendo não ter sintoma algum. Foi constatado também que pessoas mais velhas ou com condições médicas crônicas existentes, como doenças cardíacas, pulmonares, diabetes, obesidade grave, doença renal ou hepática crônica ou que tenham o sistema imunológico comprometido podem estar em maior risco de doenças graves. Nesses grupos foi observado ser mais comum que algumas pessoas apresentassem sintomas agravados, como falta de ar e pneumonia, e isso cerca de uma semana após o início dos sintomas, em muitos casos causando complicações médicas graves e podendo levar à morte algumas pessoas. Nessas complicações incluímos a pneumonia e dificuldade em respirar, falência de órgãos, problemas cardíacos, coágulos de sangue, lesão renal aguda, infecções virais e bacterianas adicionais, entre outras.

Apresentamos nesse trabalho o resultado da aplicação do projeto de ensino trabalhado em [8], que aborda uma proposta de avaliação, onde o aluno deve escrever um texto acadêmico em formato de artigo ao longo de todo o semestre, utilizando para isso as técnicas e ferramentas absorvidas ao longo do curso de Cálculo Numérico. O projeto surgiu considerando a dificuldade que um professor de Cálculo Numérico tem para fazer as avaliações da disciplina, onde geralmente envolvem questões nada práticas ou enriquecedoras e exemplos que tampouco dizem algo. Essa abordagem faz-se interessante porque o aluno espera que os resultados de suas modelagens deem resposta satisfatória para que o texto faça sentido. Além disso, o estudo dos parâmetros dos modelos traz questões totalmente enriquecedoras. O presente trabalho possivelmente requer em alguma parte os conhecimentos prévios do primeiro curso de Equações Diferenciais, porém não em todas as possíveis propostas de problemas em ecologia isso se faz necessário, como em problemas de dinâmica populacional trabalhado em [8], e em projeto sendo proposto mesmo antes do primeiro curso de Equações Diferenciais poder trazer boas respostas para análise qualitativa de problemas. Em particular, pôde ser visto que alunos que não tinham o curso de Equações Diferenciais em sua bagagem conseguiram obter um campo de visão maior do tema ao estudar exaustivamente o método de Euler. Por fim, vale a pena chamar a atenção para o interesse de alguns alunos com boa aptidão para a disciplina em continuar os estudos, levando em consideração que o projeto em si ocorre após o encerramento da disciplina, como foi em [8] e aqui. O problema apresentado no semestre em questão envolve um estudo construtivo e numérico de um problema de valor inicial central em epidemiologia, e, por fim, a aplicação de algum método de ajuste de curvas para o resultado obtido de alguma análise. Porém, desde que o Brasil foi atingido pela pandemia, a maior parte das universidades brasileiras tiveram seus períodos letivos suspensos e o projeto 
não pôde ter continuidade com os alunos correntes. Levando em consideração o fato de que um texto abordando a dinâmica de epidemias de uma forma um pouco mais acadêmica estava sendo necessário em um contexto social geral, foi decidido entre professor e uma aluna de um semestre passado a continuidade do projeto, seguindo aqui a nossa contribuição.

O presente texto foi disponibilizado para leitura e consulta dos alunos de Cálculo Numérico da Universidade do Estado de Santa Catarina do primeiro semestre de 2020. Dentre as atividades propostas, uma das mais interessantes foi a generalização do que apresentamos neste trabalho para ajustes de curvas na Seção 3.4 para o problema trabalhado em [8]: Ajustar os dados da população italiana e alemã das Figuras 22 e 23 (em [8]) ao modelo de crescimento logistico (2) (em [8]), é uma sugestão de atividade indicada pelos autores. Apresentamos de maneira formal o projeto sugerido na próxima seção.

\section{O projeto proposto}

Os vírus são um dos principais microorganismos causadores de doenças ao redor do mundo, porém não são os únicos; nesse grupo podemos incluir também as bactérias, os parasitas e os fungos. Doenças em geral causam mais mortes no mundo do que qualquer outra coisa, inclusive mais do que guerras e a fome. Com o surgimento de novas doenças ou ressurgimento das antigas torna-se cada vez mais urgente o envolvimento interdisciplinar, e a modelagem matemática dessas dinâmicas pode desempenhar uma função significativa para fazer previsões práticas e para tomadas de decisões. Modelos matemáticos para epidemias também podem ser extremamente úteis para fornecer estimativas fundamentadas do nível de vacinação para o controle de doenças infecciosas transmitidas diretamente, entre outras aplicações. Nos modelos matemáticos clássicos, os quais são relevantes ao entendimento da dinânimica de epidemias, considera-se que a população total seja constante. Se um pequeno grupo de indivíduos infectados é introduzido em uma grande população, um problema básico é descrever a propagação da infecção dentro da população em função do tempo. Obviamente, essa modelagem depende de uma imensa quantidade de variáveis, incluindo a doença real envolvida, porém em uma análise totalmente introdutória de modelar doenças transmitidas diretamente algumas suposições gerais não tão razoáveis devem ser feitas. Considere uma doença que confere imunidade em caso de recuperação, e que, se letal, inclui mortes, ou seja, os indivíduos mortos ainda são contados. Suponha que a doença seja tal que a população possa ser dividida em três classes distintas:

- os suscetíveis $\mathrm{S}$, que é a classe dos que podem pegar a doença;

- os infecciosos I, que é a classe dos que têm a doença e podem transmiti-la;

- a classe removida, R, ou seja, aqueles que tiveram a doença ou estão recuperados, imunes ou isolados até a recuperação.

Segundo [6], um modelo introdutório que relaciona as três classes acima é dado pelo sistema de equações diferenciais ordinárias

$$
\left\{\begin{array}{l}
\frac{\mathrm{dS}}{\mathrm{dt}}=-\mathrm{rSI} \\
\frac{\mathrm{dI}}{\mathrm{dt}}=\mathrm{rSI}-\mathrm{aI} \\
\frac{\mathrm{dR}}{\mathrm{dt}}=\mathrm{aI}
\end{array}\right.
$$


complementado com dados iniciais $\mathrm{S}(0)=\mathrm{S}_{0}>0$ representando a quantidade de suscetíveis à doença, $\mathrm{I}(0)=\mathrm{I}_{0}>0$ representando a quantidade inicial de infectados nessa população e $\mathrm{R}(0)=0$ representando que no instante inicial da infecção ainda não há removidos, onde ainda $\mathrm{r}>0$ é a taxa de infecção e a $>0$ é a taxa de remoção de infecciosos. Esse modelo determinístico é introdutório para entender a dinâmica básica de uma infecção, porém é deficiente em determinadas situações, como a erradicação de uma doença, pois, como exposto, a probabilidade de que os últimos indivíduos infectados infectem outro suscetível não é determinística.

Ideias que seguem desse modelo têm sido usadas para modelar a disseminação de infecções virais, bacterianas e parasitárias e até infecções fúngicas. Desse modo, a proposta é estudar o modelo e explorar numericamente suas principais propriedades, fazendo isso de maneira construtiva com discussões a respeito dos fatos, entendendo os significados dos parâmetros e identificando as limitações, verificando que é possível a inclusão de diversos efeitos relevantes e analisando qualitativamente o problema, e deixamos claro desde então que se deve ter prudência ao fazer uso prático de qualquer modelo epidêmico. Este texto foi construído dentro da disciplina de Cálculo Numérico a fim de introduzir novas ideias de avaliação dos alunos, que em sua maioria já fizeram o primeiro curso de Equações Diferenciais. Tal metodologia é interessante, uma vez que o aluno pratica a sua escrita acadêmica, complementa consideravelmente o curso de Equações Diferenciais aprimorando suas análises qualitativas com exemplos interessantes e, muitas vezes, demonstra interesse em continuar os trabalhos, mesmo após o fim da disciplina, como no presente texto.

No site da Universidade Johns Hopkins extraímos os dados apresentados nas Tabelas 1 e 2 no Apêndice B referentes a mortes, infectados e doentes por cada dia na China no período de 16 de Janeiro a 02 de Abril, onde o número de mortos e infectados já se estabilizou e deixou de ser o epicentro da pandemia. O objetivo então será o estudo do modelo (1) e algumas de suas propriedades através das ferramentas estudadas na disciplina de Cálculo Numérico e, possivelmente, comparar e fazer ajustes entre resultados numéricos e reais. Os ajustes apresentados devem ser entendidos como um passo desse caminho de absorção do modelo, sem qualquer compromisso com a realidade. Assim, o texto focará nas técnicas básicas de Cálculo Numérico a fim de entender o modelo central para epidemias proposto.

\section{Desenvolvimento do projeto}

Usaremos ao longo de todo o trabalho a ferramenta de Lista, cuja breve introdução está apresentada no Apêndice A. Em Matlab, no editor, com o auxílio das Tabelas 1 e 2 disponíveis no Apêndice B, criamos as seguintes listas:

$$
\left\{\begin{array}{l}
\text { Num_Infec }=[0,17,59,77,77,149, \cdots, 45,31,48,36,35,31] ; \\
\text { Num_Mortes }=[0,0,1,0,0,3,17, \cdots, 5,4,1,7,6,4] ; \\
\text { Num_Doentes }=[24,41,94,170,260, \cdots, 1864,1727,1562] ; \\
\mathrm{T}=[0,2,3,4,5,6,7, \cdots, 77] ;
\end{array}\right.
$$

que são os vetores com dados do período de epidemia na China, onde Num_Infec contém o número de novos infectados, Num_Mortes o número diário de mortes, Num_Doentes o número atual de doentes e $\mathrm{T}$ referente aos dias de dados colhidos da epidemia. Os comandos size(Num_Infec) ou size(Num_Mortes) retornam [1 78], que são as características de ambos os vetores, são matrizes $1 \times 78$. As mesmas catacterísticas valem para a matriz $\mathrm{T}$ de ordem $1 \times 78$ e Num_Doentes, uma matriz de ordem $1 \times 80$. A matriz de $\mathrm{T}$ pode ser criada simplesmente digitando " $\mathrm{T}=0: 1: 77$ ". A fim de plotar Num_Mortes e Num_Infect no campo dos y com T no campo dos x, utilizamos os 
comandos

$$
\begin{aligned}
& \operatorname{plot}(\mathrm{T}, \text { Num_Mortes, 'bo',' markerfacecolor',' b') } \\
& \text { plot(T, Num_Infect,' bo',' markerfacecolor', ', r'), }
\end{aligned}
$$

que retornam os resultados exibidos na Figura $1^{1}$.
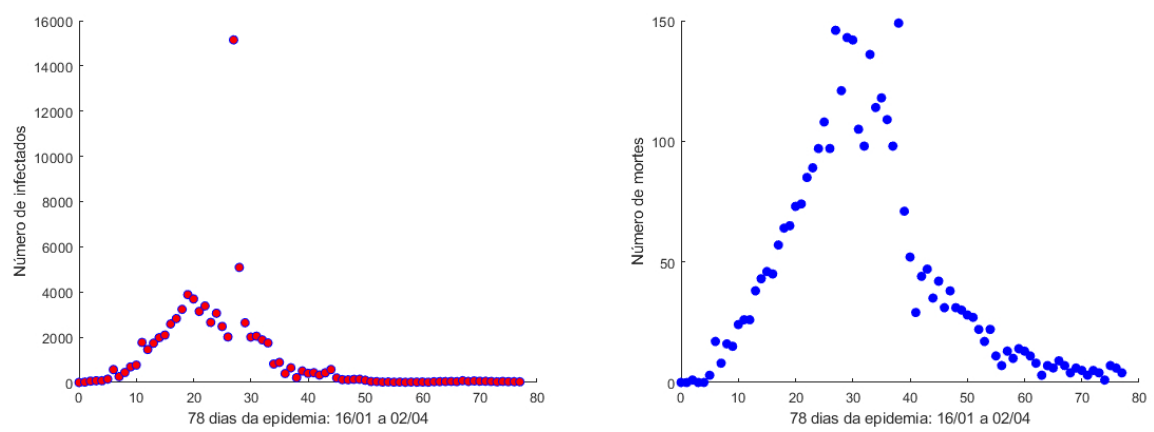

Figura 1: Plotando os dados apresentados na Tabela 1. À esquerda estão os dados dos novos infectados e à direita os dados dos novos mortos.

Com o avanço da pandemia de covid-19, um tema recorrente é o que é e quando chegará ao "pico" da infecção. Grosso modo, o pico é o dia em que o número de pessoas infectadas é igual ao número de pessoas curadas, e, a partir dele, a quantidade de pacientes recuperados começa a superar o número de novos casos e a doença começa a reduzir seu avanço. Intuitivamente, conseguimos identificá-los na Figura 1, porém, mais comum no dia a dia, e também bastante útil para o modelo que estamos estudando, é termos uma matriz com esses dados acumulados, ou seja, até o n-ésimo dia temos a soma de todos os infectados e mortos até então. Fazemos isso com um simples comando for e o auxílio dos vetores Num_Mortes e Num_Infec para criar os vetores Soma_Infec de infectados e Soma_Mortes de mortos contendo as informações acumuladas:

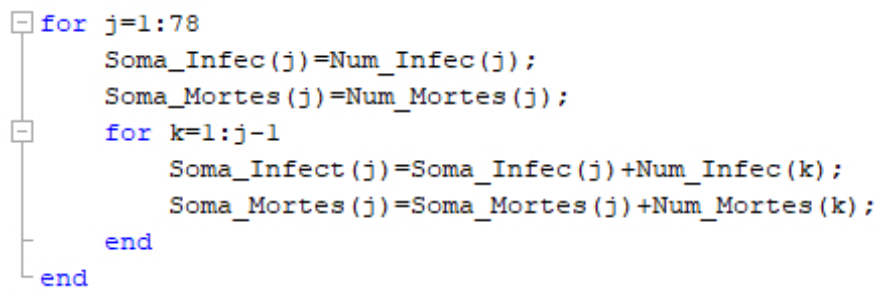

O mesmo processo para plotar Soma_Infec e Soma_Mortes dão-nos os resultados exibidos na Figura 2.

${ }^{1}$ Para várias alternativas para plotar dados como esses em Matlab sugerimos consultar os documentos e exemplos fornecidos em https://www.mathworks.com/help/matlab/ref/plot.html. 

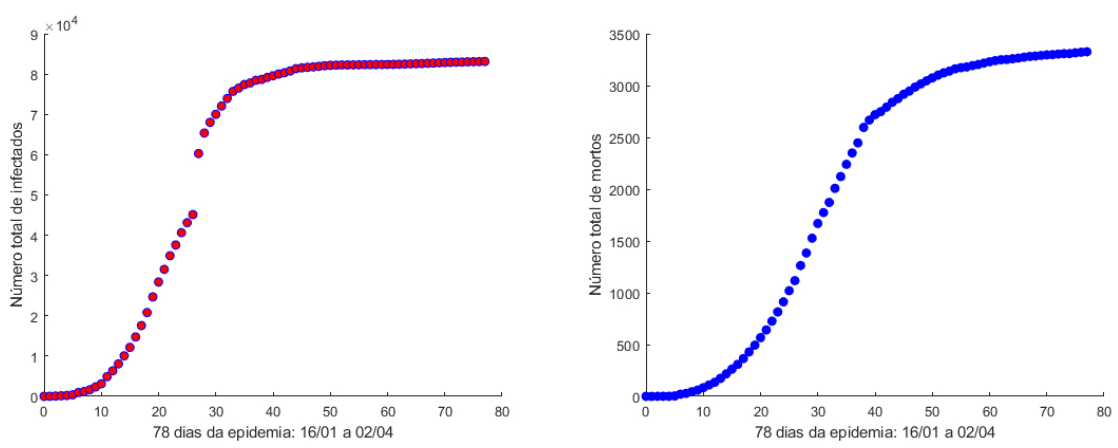

Figura 2: Plotando os dados apresentados na Tabela 1 de forma acumulada. À esquerda a quantidade de infectados e à direita a quantidade de mortos.

Discutimos brevemente os resultados das figuras 1 e 2. Na Figura 2 é apresentada a quantidade acumulada de infectados e mortos, e observamos que essas são muito baixas desde o início até aproximadamente $t=10$, e está em crecimento aparentemente exponencial até em torno de $t=30$, e então o crescimento continua, porém desacelerado, até que se aproxima de um valor limite em torno de $\mathrm{t}=50$. Funções com características como essas, quando têm derivadas, essas comportamse da seguinte maneira: São aproximadamente zero até em torno de $t=10$, são positivas e crescem quando há o crescimento exponencial e o desaceleramento do crescimento é refletido no fato de as derivadas decrescerem, porém continuarem positivas, uma vez que as funções ainda crescem. Por fim, atingir um valor limite constante, como nesse contexto, é refletido novamente no fato de a derivada dessas funções aproximarem-se de zero. Observamos que, com certo entusiasmo, o que se espera das derivadas dessas funções pode ser enxergado na Figura 1, onde é razoável imaginar que existam relações entre essas quantidades. Assim, para formalizar essas ideias, vamos entender o progresso dos indivíduos representado esquematicamente por

\section{SUSCETÍVEIS À DOENÇA $\longrightarrow$ INFECIOSOS $\longrightarrow$ MORTOS OU RECUPERADOS}

A transmissão da infecção do Covid-19 deve-se, principalmente, ao contato de pessoa para pessoa por intermédio de gotículas do nariz ou da boca as quais se espalham ao tossir, espirrar e falar. Nesse viés, quando encostamos em qualquer superfície após alguém com a doença expelir as gotículas no local, estamos sujeitos a contrair o vírus ao tocarmos nos olhos, boca ou nariz. O tempo entre a exposição do Covid-19 e o aparecimento dos sintomas, denominado período de incubação, dura em torno de 2 a 14 dias, que corresponde com a duração da quarentena recomendada. Informações sobre a transmissão da infecção e do período de incubação são cruciais em qualquer modelo epidemiológico, e essas características serão refletidas no modelo através dos termos e parâmetros. Com $\mathrm{S}(\mathrm{t})$ representando o número de suscetíveis à doença, $\mathrm{I}(\mathrm{t})$ o número de infectados e R(t) o número de removidos em cada classe no instante de tempo t, assumimos aqui que:

i Como varia a quantidade de infectados, isto é, o ganho na classe infecciosa, essa segue em uma taxa proporcional ao número de infectantes I e suscetíveis $\mathrm{S}$, isto é, rSI, em que r $>0$ é um parâmetro que vamos admitir como constante. Os suscetíveis são perdidos na mesma taxa. 
Matematicamente, escrevemos:

$$
\frac{\mathrm{dI}}{\mathrm{dt}}=\mathrm{rSI}+(\cdots) \quad \text { e } \quad \frac{\mathrm{dS}}{\mathrm{dt}}=-\mathrm{rSI} .
$$

Podemos entender as equações acima como o encontro de infectados com suscetíveis produz novos infectados, diminuindo a quantidade de suscetíveis na mesma taxa.

ii Como varia a quantidade de removidos $\mathrm{R}$, isto é, a taxa de remoção de infecciosos para a classe removida, essa segue proporcional ao número de infecciosos, ou seja, aI, onde a > 0 é uma constante; 1/a é entendido como a medida do tempo gasto no estado infeccioso. Matematicamente,

$$
\frac{\mathrm{dI}}{\mathrm{dt}}=\mathrm{rSI}-\mathrm{aI} \quad \text { e } \quad \frac{\mathrm{dR}}{\mathrm{dt}}=\mathrm{aI} .
$$

iii Supomos que o período de incubação é curto o suficiente para ser insignificante, ou seja, um suscetível que contrai a doença é infectante imediatamente.

Por fim, consideramos então as classes como uniformemente misturadas, ou seja, todo par de indivíduos tem a mesma probabilidade de entrar em contato um com o outro ${ }^{2}$. $\mathrm{O}$ modelo baseado nessas ideias é então

$$
\begin{cases}\frac{\mathrm{dS}}{\mathrm{dt}}=-\mathrm{rSI} & \text { em } \mathrm{t}>0 \operatorname{com} \mathrm{S}(0)=\mathrm{S}_{0} \\ \frac{\mathrm{dI}}{\mathrm{dt}}=\mathrm{rSI}-\mathrm{aI} & \text { em } \mathrm{t}>0 \operatorname{com} \mathrm{I}(0)=\mathrm{I}_{0} \\ \frac{\mathrm{dR}}{\mathrm{dt}}=\mathrm{aI} & \text { em } \mathrm{t}>0 \operatorname{com} \mathrm{R}(0)=0,\end{cases}
$$

onde $\mathrm{r}>0$ é a taxa de infecção e a $>0$ é a taxa de remoção de infecciosos. Esse modelo clássico foi apresentado pela primeira vez por Kermack-McKendrick em 1927. Naturalmente, estamos interessados apenas em soluções não negativas para S, I e R. Esse é um modelo básico, mas que mesmo assim nos permite fazer alguns comentários gerais altamente relevantes sobre epidemias e, de fato, descrever adequadamente algumas epidemias específicas.

Conseguimos incorporar o tamanho da população ao problema abordado no sistema (3) somando as três equações, onde obtemos

$$
\frac{\mathrm{dS}}{\mathrm{dt}}+\frac{\mathrm{dI}}{\mathrm{dt}}+\frac{\mathrm{dR}}{\mathrm{dt}}=0 \Rightarrow \mathrm{S}(\mathrm{t})+\mathrm{I}(\mathrm{t})+\mathrm{R}(\mathrm{t})=\text { Constante }
$$

onde em $\mathrm{t}=0$ sabemos que a soma da quantidade de suscetíveis, infectados e removidos é o tamanho da população total, que denotaremos por N, ou seja, Constante = N. Assim, sendo S, I e R soluções positivas de (3), essas são todas limitadas pela quantidade N. Observemos que se associarmos dados positivos $\mathrm{S}_{0}, \mathrm{I}_{0}>0$ e $\mathrm{R}(0)=0$ sempre teremos soluções positivas. De fato,

$$
\begin{aligned}
& \frac{\mathrm{dS}}{\mathrm{dt}}=-\mathrm{rSI} \Rightarrow \mathrm{S}(\mathrm{t})=\mathrm{S}_{0} \mathrm{e}^{-\mathrm{r} \int_{0}^{\mathrm{t}} \mathrm{I}(\mathrm{s}) \mathrm{ds}}>0, \\
& \frac{\mathrm{dI}}{\mathrm{dt}}=\mathrm{rSI}-\mathrm{aI} \Rightarrow \mathrm{I}(\mathrm{t})=\mathrm{I}_{0} \mathrm{e}^{\int_{0}^{\mathrm{t}} \mathrm{rS}-\mathrm{ads}}>0,
\end{aligned}
$$

\footnotetext{
${ }^{2}$ Essa é uma suposição importante e, em muitas situações, não ocorre, como na maioria das doenças sexualmente
} transmissíveis (DST) 
e $R(t)=a \int_{a}^{t} I(s) d s \geq 0$, uma vez que é a integral de uma função positiva.

O fato de uma epidemia referente a uma nova doença ter começado com um vírus que já era conhecido mas até então não apresentava problemas com contaminações fez com que os dados que eram apresentados diariamente ficassem confusos. Obviamente, pelo que temos exposto na tabela, a epidemia não começou ali, e muito se discute o início da mesma. A fim de aproximar a curva dos removidos $\mathrm{R}(\mathrm{t})$, utilizamos, finalmente os dados do vetor Num_Doentes combinado com o seguinte raciocínio:

$$
\text { Doentes hoje }=\text { Doentes ontem }- \text { Recuperados ou mortos }+ \text { Novos infectados }
$$

Então podemos escrever

$$
\text { Doentes hoje }- \text { Doentes ontem }=- \text { Recuperados ou mortos }+ \text { Novos infectados }
$$

Somando essas duas quantidades, e denotando

$$
\begin{aligned}
& \mathbf{R}(\mathrm{t})=\text { Soma dos recuperados ou mortos até o dia } \mathrm{t} \\
& \text { Soma_Infec }(\mathrm{t})=\text { Soma dos novos infectados até o dia } \mathrm{t} \\
& \Delta \mathrm{d}(\mathrm{t})=\text { Soma das diferenças diárias dos doentes até o dia } \mathrm{t},
\end{aligned}
$$

vamos obter

$$
\Delta \mathrm{d}(\mathrm{t})=-\mathbf{R}(\mathrm{t})+\text { Soma_Infec }(\mathrm{t}),
$$

donde vamos aproximar $\mathbf{R}(\mathrm{t})=$ Soma_Infec $(\mathrm{t})-\Delta \mathrm{d}(\mathrm{t})$. Plotamos da mesma forma como acima, à esquerda na Figura 3, o vetor Num_Doentes, e podemos ver que a quantidade $\Delta \mathrm{d}(\mathrm{t})$ pode ser positiva, negativa ou zero, porém podemos estimar que $\mathrm{R}(\mathrm{t})$ deve respeitar a relação

$$
\text { Soma_Infec(t) }-|\Delta d(t)| \leq R(t) \leq \text { Soma_Infec(t) }+|\Delta d(t)| .
$$

Assim, seguindo as mesmas ideias para obter Soma_Mortes e Soma_Infec, criamos os vetores contendo "Soma1 $=$ Soma_Infec $-\Delta \mathrm{d}(\mathrm{t})$ " e "Soma2 = Soma_Infec $+\Delta \mathrm{d}(\mathrm{t})$ " e plotamos.
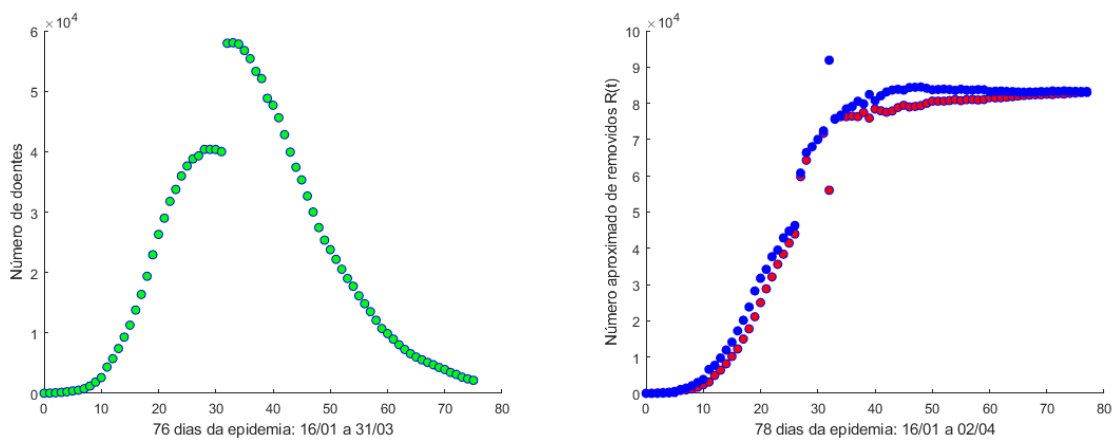

Figura 3: $\grave{A}$ esquerda o número de doentes infecciosos $\mathrm{I}(\mathrm{t})$ e à direita os dados do vetor Soma1 em vermelho e Soma2 em azul dando uma ideia para a curva $\mathrm{R}(\mathrm{t})$.

Finalmente, então, na Figura 3 temos a ideia da curva solução I(t) e R(t) que devemos encontrar. Ao longo de todo trabalho, usaremos a notação $\mathrm{S}(\mathrm{t}), \mathrm{R}(\mathrm{t})$ e I(t) para as soluções analíticas do sistema 3 e $\mathbf{S}, \mathbf{R}$ e I para quaisquer que sejam os dados discretos, com a óbvia relação entre eles. 


\subsection{Aplicação do método de Euler para (3)}

A fim de obter a solução numérica para (3), utilizaremos o método de Euler.

Entenderemos por solução numérica de um problema de valor inicial com um sistema de equações diferenciais ordinárias como (3) como uma quantidade de pontos $\left(t_{i}, S_{i}\right),\left(t_{i}, I_{i}\right)$, $\left(\mathrm{t}_{\mathrm{i}}, \mathrm{R}_{\mathrm{i}}\right)$ com $\mathrm{i}=1,2, \cdots, \mathrm{n}$, de modo que uma poligonal ligando pontos adjacentes dê a aproximação da solução exata do problema.

O método de Euler pode ser visto com detalhes em [2], apresentamos aqui ligeiramente. Consideremos $\mathrm{Y}$ uma função diferenciável. Se $\mathrm{h} \in \mathbb{R}, \mathrm{h} \neq 0$, é um valor pequeno em magnitude, uma boa aproximação para a derivada de $\mathrm{Y}$ em um instante de tempo t é dada por

$$
\mathrm{Y}^{\prime}(\mathrm{t}) \approx \frac{\mathrm{Y}(\mathrm{t}+\mathrm{h})-\mathrm{Y}(\mathrm{t})}{\mathrm{h}} .
$$

A igualdade vale no limite de $\mathrm{h}$ tendendo a zero. Suponhamos nesse caso $\mathrm{h}>0$. A discretização (5) pode ser entendida por

$$
\mathrm{Y}^{\prime}(\mathrm{t}) \approx \frac{\mathrm{Y}(\mathrm{t}+\mathrm{h})-\mathrm{Y}(\mathrm{t})}{\mathrm{h}}=\frac{\mathrm{Y} \text { avaliado um passo à frente }-\mathrm{Y} \text { atual }}{\mathrm{h}} .
$$

Então, o primeiro passo para obter uma solução numérica de (3) em um intervalo de tempo $\left[0, \mathrm{~T}_{\text {final }}\right]$, onde $\mathrm{T}_{\text {final }}>0$ é um valor a ser escolhido, é considerar uma grade de tempos $\mathrm{T}=$ $\left[\mathrm{t}_{1}, \mathrm{t}_{2} \cdots, \mathrm{t}_{\mathrm{n}}\right]$, onde $\mathrm{t}_{1}=0, \mathrm{t}_{\mathrm{i}+1}=\mathrm{t}_{\mathrm{i}}+\mathrm{h}$ para $\mathrm{i}=1, \cdots, \mathrm{n}-1$, com $\mathrm{t}_{\mathrm{n}}=\mathrm{T}_{\text {final }}$. Assim, $\mathrm{n}$ e $\mathrm{h}$ são relacionados por $\mathrm{h}=\mathrm{T}_{\text {final }} / \mathrm{h}$. Denotamos então $\mathbf{S}_{\mathrm{i}}=\mathbf{S}\left(\mathrm{t}_{\mathrm{i}}\right), \mathbf{I}_{\mathrm{i}}=\mathbf{I}\left(\mathrm{t}_{\mathrm{i}}\right)$ e $\mathbf{R}_{\mathrm{i}}=\mathbf{R}\left(\mathrm{t}_{\mathrm{i}}\right)$. Da mesma forma como procedemos nas figuras 1 e 2, plotaremos os vetores $\mathbf{S}, \mathbf{I}$ e $\mathbf{R}$ no eixo dos y com $\mathrm{T}$ no eixo dos $\mathrm{x}$, onde

$$
\left\{\mathbf{S}=\left[\mathrm{S}_{1}, \mathrm{~S}_{2}, \mathrm{~S}_{3}, \mathrm{~S}_{4}, \cdots, \mathrm{S}_{\mathrm{n}}\right], \quad \mathbf{I}=\left[\mathbf{I}_{1}, \mathbf{I}_{2}, \mathbf{I}_{3}, \mathbf{I}_{4}, \cdots, \mathbf{I}_{\mathrm{n}}\right], \quad \mathbf{R}=\left[\mathbf{R}_{1}, \mathbf{R}_{2}, \mathbf{R}_{3}, \mathbf{R}_{4}, \cdots, \mathbf{R}_{\mathrm{n}}\right],\right.
$$

onde já sabemos que $\mathbf{S}_{1}=\mathrm{S}(0), \mathbf{I}_{1}=\mathrm{I}(0)$ e $\mathbf{R}_{1}=\mathrm{R}(0)=0$. A aplicação de (5) para o sistema (3) segue abaixo:

$$
\begin{array}{cll}
\frac{\mathrm{d} S}{\mathrm{dt}}=-\mathrm{rSI} & \Rightarrow & \frac{\mathbf{S}_{\mathrm{i}+1}-\mathbf{S}_{\mathrm{i}}}{\mathrm{h}}=-\mathrm{r} \mathbf{S}_{\mathrm{i}} \mathbf{I}_{\mathrm{i}} \\
\frac{\mathrm{dI}}{\mathrm{dt}}=\mathrm{rSI}-\mathrm{aI} & \Rightarrow & \frac{\mathbf{I}_{\mathrm{i}+1}-\mathbf{I}_{\mathrm{i}}}{\mathrm{h}}=\mathrm{r} \mathbf{S}_{\mathrm{i}} \mathbf{I}_{\mathrm{i}}-\mathrm{a} \mathbf{I}_{\mathrm{i}} \\
\frac{\mathrm{dR}}{\mathrm{dt}}=\mathrm{aI} & \Rightarrow & \frac{\mathbf{R}_{\mathrm{i}+1}-\mathbf{R}_{\mathrm{i}}}{\mathrm{h}}=\mathrm{a} \mathbf{I}_{\mathrm{i}},
\end{array}
$$

para $\mathrm{i}=1,2, \cdots, \mathrm{n}=\mathrm{T}_{\text {final }} / \mathrm{h}$, donde obtemos o algoritmo

$$
\left\{\mathbf{S}_{\mathrm{i}+1}=\mathbf{S}_{\mathrm{i}}-\mathrm{hr} \mathbf{S}_{\mathrm{i}} \mathbf{I}_{\mathrm{i}}, \quad \mathbf{I}_{\mathrm{i}+1}=\mathbf{I}_{\mathrm{i}}-\mathrm{hr} \mathbf{S}_{\mathrm{i}} \mathbf{I}_{\mathrm{i}}-\mathrm{ha} \mathbf{I}_{\mathrm{i}} \quad \mathbf{R}_{\mathrm{i}+1}=\mathbf{R}_{\mathrm{i}}-+ \text { ha } \mathbf{I}_{\mathrm{i}}\right.
$$


para $\mathrm{i}=1, \cdots, \mathrm{n}$, com $\mathbf{S}_{1}=\mathrm{S}(0), \mathbf{I}_{1}=\mathrm{I}(0)$ e $\mathbf{R}_{1}=0$. Para implementar (7), em Matlab, digitamos

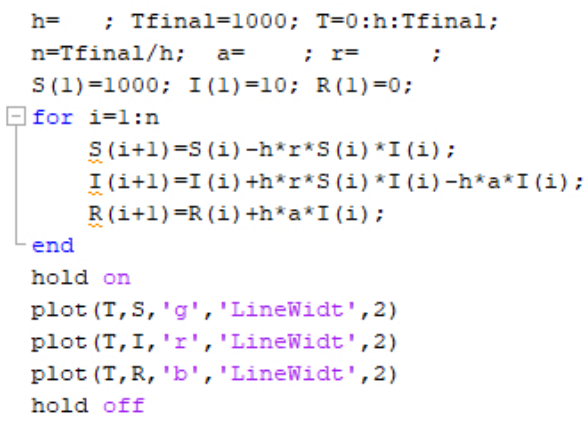

onde para os experimentos abaixo escolhemos $\mathrm{T}_{\text {final }}=1000, \mathrm{~h}=0.1$, os dados iniciais em (3) sendo $\mathrm{S}_{0}=1000$ e $\mathrm{I}_{0}=10$, e testamos alguns parâmetros para a e r. Na Figura 4, fixamos o parâmetro $\mathrm{r}=0.00005$ e testamos algumas variações para a. Da mesma forma, na Figura 5 fixamos o parâmetro $\mathrm{a}=0.02$ e testamos a sensibilidade do parâmetro $\mathrm{r}$. Em ambos os casos teremos em verde a solução numérica para $\mathrm{S}$, em vermelho a solução numérica para I e em azul a solução numérica para R. Em ambos os casos, podemos notar com esses experimentos numéricos que $\lim _{\mathrm{t} \rightarrow+\infty} \mathrm{I}(\mathrm{t})=0$, enquanto que

$$
S(\infty)=\lim _{t \rightarrow+\infty} S(t) \quad \text { e } R(\infty):=\lim _{t \rightarrow+\infty} R(t)
$$

são desconhecidos e precisam ser estudados por representar valores interessantes para a dinâmica. No primeiro experiemento na Figura 4, com $\mathrm{r}$ fixo, podemos ver que o parâmetro a influencia diretamente na curva de infectados $\mathrm{I}(\mathrm{t})$, pequenas variações para cima achatam a solução, o que era esperado, uma vez que o parâmetro a indica a que taxa os infectados migram para a condição de removidos.

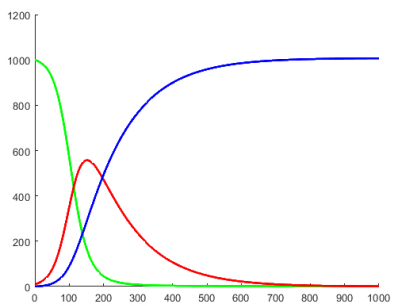

$\mathrm{a}=0.008$ e $\mathrm{r}=0.00005$.

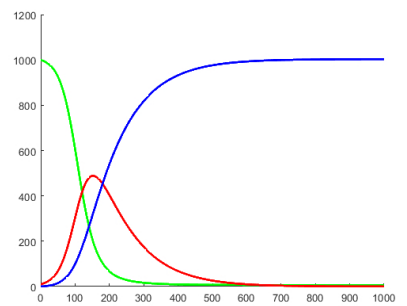

$\mathrm{a}=0.01$ e $\mathrm{r}=0.00005$.

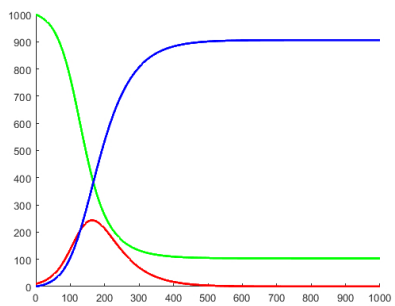

$\mathrm{a}=0.02$ e $\mathrm{r}=0.00005$. 


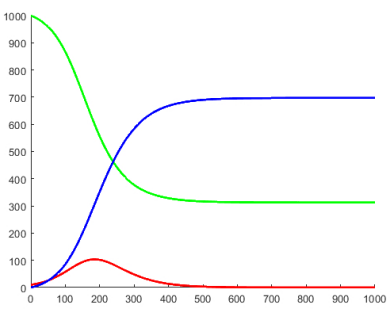

$\mathrm{a}=0.03$ e $\mathrm{r}=0.00005$.

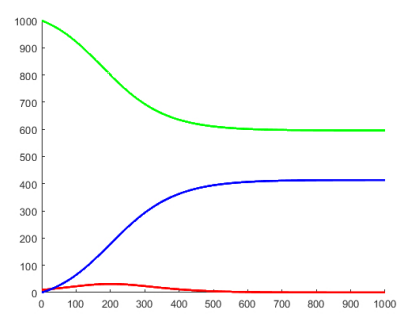

$\mathrm{a}=0.04$ e $\mathrm{r}=0.00005$

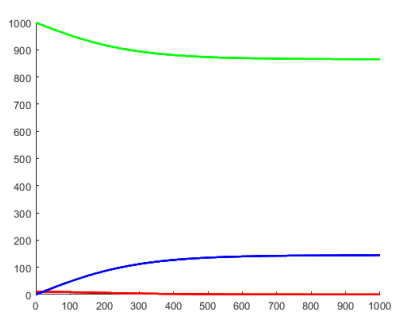

$\mathrm{a}=0.05$ e $\mathrm{r}=0.00005$.

Figura 4: Resultado do primeiro experimento com dados $\mathbf{S}(1)=1000 ; \mathbf{I}(1)=10 ; \mathbf{R}(1)=0$ e os parâmetros a e r como na imagem.

Da mesma forma, no segundo experimento, ao fixar o parâmetro a e testar r, observamos que pequenas variações para cima refletem no achatamento da curva de infectados I(t), parâmetros maiores sugerem que essa curva fica menos achatada e os valores limite $S(\infty)$ decrescem e $R(\infty)$ crescem, respeitando a relação $S(\infty)+R(\infty)=N$.

Os esforços para conter completamente a disseminação do novo coronavírus no mundo falharam, os casos registrados no mundo rapidamente iniciaram o crescimento exponencial devido à alta taxa de infecção do vírus, indicando o descontrole. Após a estabilização da epidemia na China, a Itália passou a ser o epicentro da pandemia, onde em alguns locais houve a resistência ao isolamento, e o mundo viu a rápida taxa de crescimento do vírus no país lotar alguns hospitais, forçando as salas de emergência a fecharem suas portas para novos pacientes, contratar centenas de novos médicos e solicitar suprimentos de emergência de equipamentos médicos básicos do exterior, como máscaras e respiradores. Essa falta de recursos contribuiu, em parte, para a enorme taxa de mortalidade do Covid-19 na Itália, que foi aproximadamente 7\%. Isso posto, mostra-se que há diferenca na taxa de infecção de uma população se há leitos hospitalares suficientes, profissionais e recursos para tratar os doentes. Nesse viés, tal fato recorreu a atenção do mundo e a expressão "achatamento da curva" ficou bastante comum. Na epidemiologia, "achatamento a curva" é a ideia de diminuir a propagação de um vírus para que menos pessoas precisem procurar tratamento, e para tal, necessita-se da implementação de diretrizes de distanciamento social. Assim, como podemos ver nos experimentos, variações na taxa de infecção dão-nos curvas de infecção como na Figura 6.

A geometria da curva assume formas diferentes de acordo com a taxa de infecção $r$ do vírus, podendo essa ser acentuada, na qual o vírus espalha-se exponencialmente e o número total de casos dispara até o pico dentro de algumas semanas, e, após, apresentam uma queda acentuada depois que o vírus infecta praticamente todos os suscetíveis. Quanto mais rápida a curva de infecção aumenta, mais rápido o sistema de saúde local fica sobrecarregado, como vimos na Itália, onde cada vez mais novos pacientes foram forçados a ficar sem leitos de UTI, e gradativamente os hospitais tiveram que ficar sem os suprimentos básicos necessários para conter o surto. Quando os esforços para o achatamento da curva dão resposta, pressupõe-se que haverá o mesmo número de pessoas infectadas, porém em um espaço de tempo maior, deixando o sistema de saúde menos sobrecarregado e menos pessoas doentes sendo afastadas de suas atividades. 


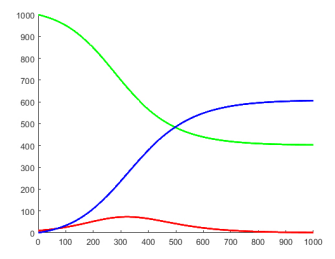

$\mathrm{a}=0.02$ e $\mathrm{r}=0.00003$.

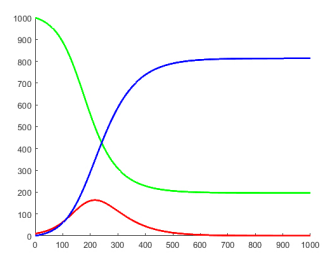

$\mathrm{a}=0.02$ e $\mathrm{r}=0.00004$.

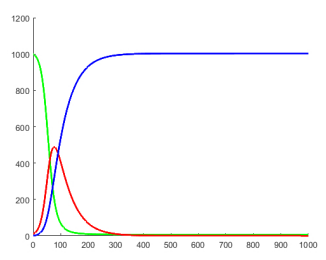

$\mathrm{a}=0.02$ e $\mathrm{r}=0.0001$.

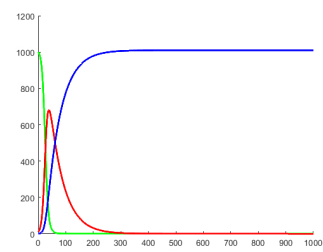

$\mathrm{a}=0.02$ e $\mathrm{r}=0.0002$.

Figura 5: Resultado do experimento para $\mathbf{S}(1)=1000 ; \mathbf{I}(1)=10 ; \mathbf{R}(1)=0$ e os parâmetros a e r como na imagem.

Atualmente, não há vacina ou medicamento especifico para tratar Covid-19 e os testes são muito limitados, por conseguinte, os métodos para achatar a curva são feitos por meio de ações coletivas. Foram feitas recomendações em todo o mundo para que as pessoas lavassem as mãos com frequência, se isolassem quando estivessem doentes ou suspeitassem que estivessem, e começassem o "distanciamento social", que essencialmente consiste em evitar outras pessoas sempre que possível. Para cumprir tais medidas, os Estados fecharam temporariamente escolas e universidades, determinaram que eventos em geral fossem cancelados ou adiados, muitas empresas adotaram o home office, entre outras várias medidas.

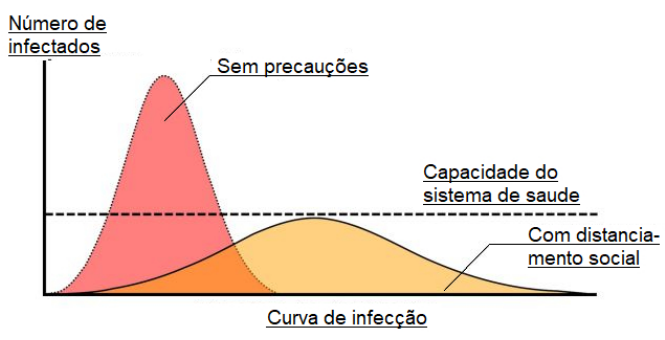

Figura 6: Adaptada de https://coronavirus.jhu.edu/map.html

Por fim, um outro detalhe dos experimentos numéricos apresentados que vale a pena discutir é o valor $\mathrm{R}(\infty)$, que indica a quantidade de pessoas que não foram infectadas. Dedicaremos a próxima seção para esse estudo, onde estudaremos o plano de fase do sistema (3).

\subsection{Aplicação de zero de funções para obter $S(\infty)$ e $R(\infty)$}

O termo epidemia, à luz do que temos apresentado, significa que para algum instante de tempo após o início da dinâmica tenha-se o número de infectados maior do que no início, que matematicamente quer dizer que existe $\mathrm{t}>0$ de modo que $\mathrm{I}(\mathrm{t})>\mathrm{I}_{0}$. Como vimos nos exemplos numéricos abordados anteriormente, dados $\mathrm{r}$, a, $\mathrm{S}_{0}$ e o número inicial de infecciosos $\mathrm{I}_{0}$, uma questão-chave em qualquer situação epidêmica é saber se a infecção se espalhar-se-á ou não, e, caso ocorra, como se desenvolve com o tempo e quando começará a declinar. Esse raciocínio tem como eixo a equação da população 
de infectados I, onde a partir de sua equação de (3), segue que

$$
\frac{\mathrm{dI}}{\mathrm{dt}}(\mathrm{t})=\mathrm{I}(\mathrm{t})(\mathrm{rS}(\mathrm{t})-\mathrm{a}) \stackrel{\mathrm{t}=0}{\Rightarrow} \quad \frac{\mathrm{dI}}{\mathrm{dt}}(0)=\mathrm{I}_{0}\left(\mathrm{rS}_{0}-\mathrm{a}\right) .
$$

Estamos interessados em saber o sinal da derivada de I para ter informações a respeito de seu crescimento. Pela equação acima verificamos rapidamente que $\frac{\mathrm{dI}}{\mathrm{dt}}(0)=0$ se $\mathrm{S}_{0}=\mathrm{a} / \mathrm{r}$, onde, claramente, estamos assumindo todos os coeficientes e dados iniciais sendo estritamente positivos. Do mesmo modo, nos casos $\mathrm{S}_{0}<\mathrm{a} / \mathrm{r}$ temos $\frac{\mathrm{dI}}{\mathrm{dt}}(0)>0$ e $\mathrm{S}_{0}>\mathrm{a} / \mathrm{r}$ segue $\frac{\mathrm{dI}}{\mathrm{dt}}(0)<0$. Da mesma forma, via a equação de suscetíveis à infecção $\mathrm{S}$ em $(3)$, temos que $\frac{\mathrm{dS}}{\mathrm{dt}}(\mathrm{t}) \leq 0$, ou seja, $\mathrm{S}(\mathrm{t})$ é não crescente e podemos concluir rapidamente que $\mathrm{S}(\mathrm{t}) \leq \mathrm{S}_{0}$. Assim, se tivermos $\mathrm{S}_{0}<\mathrm{a} / \mathrm{r}$, então

$$
\frac{\mathrm{dI}}{\mathrm{dt}}=\mathrm{I}(\mathrm{rS}-\mathrm{a}) \leq \mathrm{I}\left(\mathrm{rS}_{0}-\mathrm{a}\right)=\operatorname{Ir}\left(\mathrm{S}_{0}-\mathrm{a} / \mathrm{r}\right) \leq 0
$$

para todo $\mathrm{t} \geq 0$, e nesse caso $\mathrm{I}_{0}>\mathrm{I}(\mathrm{t}) \rightarrow 0$ quando $\mathrm{t} \rightarrow+\infty$, e assim a infecção desaparece sem que ocorra a epidemia. É fácil ver analiticamente que esse limite, de fato, é zero. Integrando a terceira equação de (3) em [0,t], temos que

$$
\int_{0}^{\mathrm{t}} \mathrm{I}(\mathrm{t}) \mathrm{dt}=\frac{1}{\mathrm{a}} \int_{0}^{\mathrm{t}} \frac{\mathrm{dR}}{\mathrm{dt}} \mathrm{dt}=\frac{1}{\mathrm{a}}(\mathrm{R}(\mathrm{t})-\mathrm{R}(0))=\frac{\mathrm{R}(\mathrm{t})}{\mathrm{a}} .
$$

Como $\mathrm{R}(\mathrm{t}) \leq \mathrm{N}$, temos que o limite de $\mathrm{t} \rightarrow+\infty$ garante que I é integrável em $[0,+\infty)$, e sua continuidade garante que $\mathrm{I}(\mathrm{t}) \rightarrow 0$ quando $\mathrm{t} \rightarrow+\infty$. Quando $\mathrm{S}_{0}>\mathrm{a} / \mathrm{r}$ segue que $\mathrm{I}(\mathrm{t})$ inicialmente cresce e então temos a epidemia. Portanto, existe um intervalo no qual o dado inicial $\mathrm{S}_{0}$ deve pertencer para que haja ou não a epidemia. Resumidamente, se $S_{0}>a / r$ temos a epidemia presente, enquanto que se $\mathrm{S}_{0}<\mathrm{a} / \mathrm{r}$ não há epidemia. Daqui para frente denotaremos os parâmetros críticos $\rho:=\mathrm{a} / \mathrm{r}$ e $\sigma=1 / \rho=\mathrm{r} / \mathrm{a}$, que são respectivamente chamados de taxa de remoção relativa e taxa de contato de infecções. Denotamos também

$$
\mathrm{R}_{0}=\frac{\mathrm{rS}_{0}}{\mathrm{a}}
$$

que representa a taxa básica de reprodução da infecção, ou seja, o número de infecções secundárias produzidas por uma infecção primária em uma população totalmente suscetível. Aqui 1/a pode ser entendido como o período infeccioso médio. Se mais de uma infecção secundária é produzida a partir de uma infecção primária, ou seja, $\mathrm{R}_{0}>1$, ocorre claramente uma epidemia. Todas essas questões envolvendo os limiares nas epidemias são obviamente importantes, e, em estudos mais aprofundados, a definição ou cálculo da taxa básica de reprodução é crucial e pode ser bastante complexo. A taxa básica de reprodução é um agrupamento crucial de parâmetros para lidar com uma epidemia ou simplesmente uma doença que está atualmente sob controle com a vacinação, por exemplo. Embora, como apresentaremos a seguir, os argumentos sejam baseados em $\mathrm{R}_{0}$, eles são bastante gerais. Com clareza, uma maneira de reduzir a taxa de reprodução $\mathrm{R}_{0}$ é reduzir o número de suscetíveis, $\mathrm{S}_{0}$.

Podemos obter alguns resultados analíticos úteis das duas primeiras equações de (3). Vale a pena reparar que a equação de $\mathrm{R}$ não é acoplada ao sistema. Note que para obter o plano de fase ${ }^{3}$ temos

$$
\frac{\mathrm{dI}}{\mathrm{dS}}=-\frac{(\mathrm{rS}-\mathrm{a}) \mathrm{I}}{\mathrm{rSI}}=-1+\frac{\rho}{\mathrm{S}},
$$

${ }^{3}$ Veja [1], por exemplo. Em [6] pode ser visto uma revisão bastante interessate e completa. 
onde estamos supondo $I \neq 0$. As singularidades ocorrem quando $I=0$. Integrando essa equação, temos simbolicamente que

$$
\int \mathrm{dI}=\int-1+\frac{\rho}{\mathrm{S}} \mathrm{d} \mathrm{S},
$$

donde obtemos as trajetórias do plano de fase

$$
\mathrm{I}+\mathrm{S}-\rho \ln (\mathrm{S})=\text { constante. }
$$

Assim, essa equação é constante para cada valor de $t$, tomando $t=0$, obtemos

$$
\mathrm{I}+\mathrm{S}-\rho \ln (\mathrm{S})=\mathrm{I}_{0}+\mathrm{S}_{0}-\rho \ln \left(\mathrm{S}_{0}\right) .
$$

Essas curvas podem ser obtidas numericamente plotando os vetores S por I obtidos em cada experimento acima, e para isso completaremos o algoritmo dos experimentos anteriores com as linhas

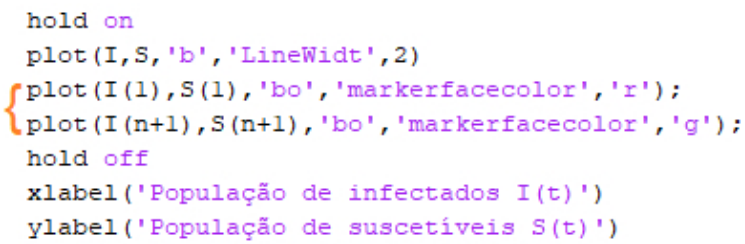

Essas linhas dar-nos-ão uma curva em azul (I(t), S(t)) começando no ponto ( $(0)$, S(0)) em vermelho e terminando em um ponto verde ainda a ser estudado; esses dois pontos são plotados como destacado na chave vermelha. O que sabemos sobre o ponto vermelho é que ele sempre está no primeiro quadrante e sobre a reta $\mathrm{y}(\mathrm{t})=\mathrm{N}-\mathrm{t}$, e isso se reflete do fato de que $\mathrm{S}(0)+\mathrm{I}(0)=\mathrm{N}$ e $R(0)=0$. Apresentamos na Figura 7 dois exemplos de planos de fase. Faremos a leitura da seguinte forma: O ponto vermelho representa o par de dados iniciais $(\mathrm{I}(0), \mathrm{S}(0))$ e essa curva segue para o ponto verde, onde sabemos a coordenada do eixo $\mathrm{x}$, uma vez que $\mathrm{I}(\infty)=0$, e temos a leitura de que, em ambos os exemplos, a infecção I(t) cresce com o tempo e, em algum determinado instante, $\mathrm{I}(\mathrm{t})$ passa a decrescer e vai para zero enquanto que $\mathrm{S}(\mathrm{t})$ decresce em toda a dinâmica.
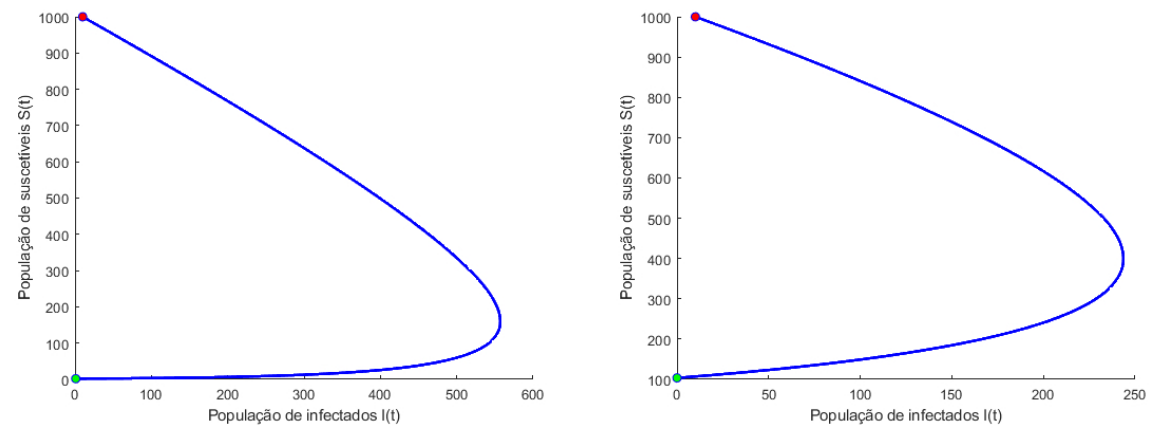

Figura 7: Plano de fase para $(\mathrm{a}, \mathrm{r})=(0.008,0.00005)$ à esquerda $e(\mathrm{a}, \mathrm{r})=(0.02,0.00005)$ à direita. Os dados iniciais também são os mesmos do experimento, isto é, $\mathrm{S}(0)=1000$ e $\mathrm{I}(0)=10$. 
Se acontece o fenômeno da epidemia um dos principais interesses é saber o quão severa ela é. Pela equação de I em (3), temos que I deve ter máximo onde $\frac{\mathrm{dI}}{\mathrm{dt}}=0$, e isso acontece no instante de tempo t em que a população dos suscetíveis $\mathrm{S}$ é tal que $\mathrm{S}=\rho$. Assim, $\mathrm{S}=\rho$ em (8) implica que

$$
\begin{aligned}
\mathrm{I}_{\max } & =\rho \ln (\rho)-\rho+\mathrm{I}_{0}+\mathrm{S}_{0}-\rho \ln \left(\mathrm{S}_{0}\right) \\
& =\mathrm{I}_{0}+\mathrm{S}_{0}-\rho+\rho \ln \left(\frac{\rho}{\mathrm{S}_{0}}\right) \\
& =\mathrm{N}-\rho+\rho \ln \left(\frac{\rho}{\mathrm{S}_{0}}\right) .
\end{aligned}
$$

Portanto, temos um ponto a ser destacado na curva do plano de fase, que é o ponto $\left(\mathrm{I}_{\max }, \rho\right)$ onde a infecção atinge seu máximo. Completamos as linhas em Matlab para

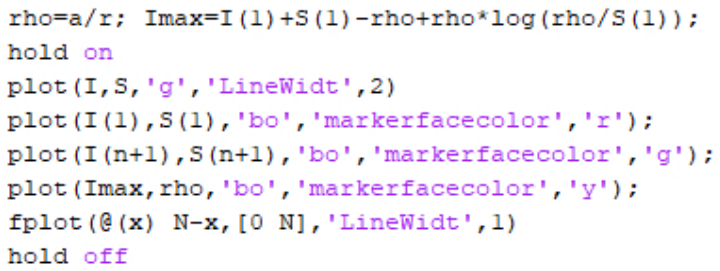

onde acrescentamos o ponto $\left(\mathrm{I}_{\max }, \rho\right)$ em amarelo e o gráfico da reta $\mathrm{y}(\mathrm{t})=\mathrm{N}-\mathrm{t}$, onde lembramos que $\mathrm{N}$ é o número total da população, que nos experimentos acima tomamos como sendo 1010. Usamos isso para o experimento apresentado na Figura 8, onde compilamos algumas vezes com parâmetros fixos $\mathrm{S}(0)=700, \mathrm{I}(0)=310, \mathrm{a}=0.018$ e $\mathrm{r}$ variando como na legenda.

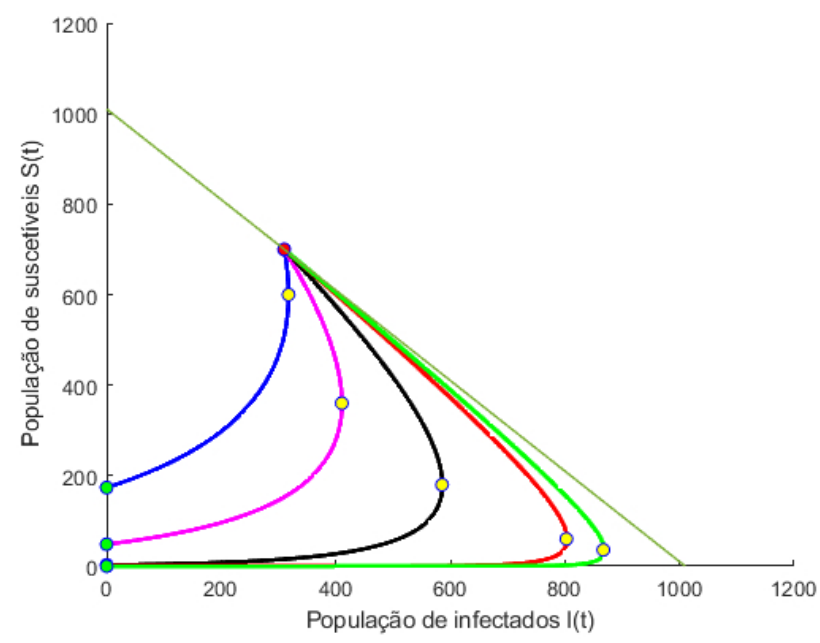

Figura 8: Experimento realizado com dados $\mathrm{S}(0)=700, \mathrm{I}(0)=310, \mathrm{a}=0.018$ e $\mathrm{r}=0.00003$ (azul), 0.00005 (rosa), 0.0001 (preto), 0.0003 (vermelho), 0.0005 (verde).

Para dados iniciais $\mathrm{I}_{0}$ e $\mathrm{S}_{0}>\rho$ sabemos que a epidemia ocorre e o plano de fase começa com $\mathrm{S}>\rho$ e podemos ver que I cresce para $\mathrm{I}_{0}$ e então uma epidemia segue. Essa pode não ser uma epidemia 
tão severa no caso de o dado inicial $\mathrm{I}_{0}$ ser próximo ao valor $\mathrm{I}_{\max }$, como podemos ver nas curvas em azul e rosa, por exemplo. Ainda, se $\mathrm{S}_{0}<\rho$, então a epidemia não ocorre e temos simplesmente I decrescedo para 0 . O eixo $\mathrm{I}=0$ é uma linha de singularidades e em todas as trajetórias já concluímos que $\mathrm{I}(\mathrm{t}) \rightarrow 0$ quando $\mathrm{t} \rightarrow+\infty$. Pela equação de $\mathrm{S}$ em $(3)$, temos que $\mathrm{S}$ decresce uma vez que $\frac{d \mathrm{~S}}{\mathrm{dt}}<0$ para $\mathrm{S} \neq 0$ e $\mathrm{I} \neq 0$. Procedendo da mesma forma para as primeira e segunda equação de (3), segue que

$$
\begin{aligned}
\frac{\mathrm{dS}}{\mathrm{dR}}=-\frac{\mathrm{S}}{\rho} & \Rightarrow \quad \mathrm{S}=\mathrm{S}_{0} \mathrm{e}^{-\mathrm{R} / \rho} \geq \mathrm{S}_{0} \mathrm{e}^{-\mathrm{N} / \rho}>0 \\
& \Rightarrow \quad 0<\mathrm{S}(\infty) \leq \mathrm{N} .
\end{aligned}
$$

De fato, ainda pela primeira equação de (3), temos que

$$
\begin{array}{rlrl}
\frac{\mathrm{dI}}{\mathrm{dt}}=-\mathrm{aIS} & \Rightarrow \mathrm{S}(\mathrm{t})=\mathrm{S}(0) \mathrm{e}^{-\mathrm{r} \int_{0}^{\mathrm{t}} \mathrm{I}(\mathrm{s}) \mathrm{ds}} \\
& \Rightarrow \mathrm{S}(\mathrm{t})=\mathrm{S}_{0} \mathrm{e}^{-\frac{\mathrm{r}}{\mathrm{a}} \mathrm{R}(\mathrm{t})} \\
& \Rightarrow & \mathrm{S}(\mathrm{t})=\mathrm{S}_{0} \mathrm{e}^{-\mathrm{R}(\mathrm{t}) / \rho} .
\end{array}
$$

Também, como $\mathrm{I}(\infty)=0$, tem-se que

$$
\begin{array}{lll}
\mathrm{S}(\mathrm{t})+\mathrm{I}(\mathrm{t})+\mathrm{R}(\mathrm{t})=\mathrm{N} & \stackrel{\mathrm{t} \rightarrow+\infty}{\Rightarrow} & \mathrm{S}(\infty)+\mathrm{R}(\infty)=\mathrm{N} \\
& \Rightarrow & \mathrm{R}(\infty)=\mathrm{N}-\mathrm{S}(\infty) .
\end{array}
$$

Suponhamos então que estamos no caso $\mathrm{S}_{0}<\rho$ onde há a epidemia. Assim, por (10) e (11), temos que

$$
\mathrm{S}(\infty)=\mathrm{S}_{0} \mathrm{e}^{-\mathrm{R}(\infty) / \rho}=\mathrm{S}_{0} \mathrm{e}^{-(\mathrm{N}-\mathrm{S}(\infty)) / \rho},
$$

e portanto $\mathrm{S}(\infty)$ é a raiz positiva da equação $\mathrm{S}_{0} \mathrm{e}^{-\frac{\mathrm{N}-\mathrm{s}}{\rho}}=\mathrm{s}$, com $0<\mathrm{s}<\rho$. Obtemos o número total $\mathrm{I}_{\text {total }}$ de suscetíveis que pegam a doença no curso da epidemia por

$$
\mathrm{I}_{\text {total }}=\mathrm{I}_{0}+\mathrm{S}_{0}-\mathrm{S}(\infty)
$$

onde $S(\infty)$ é determinada como discutido acima. Uma implicação importante dessa análise, a saber, é que $\mathrm{I}(\mathrm{t}) \rightarrow 0$, e $\mathrm{S}(\mathrm{t}) \rightarrow \mathrm{S}(\infty)>0$, é que a doença morre por falta de infecciosos e não por falta de suscetíveis.

Seja $\mathrm{f}: \mathbb{R} \rightarrow \mathbb{R}$ uma função dada. A fim de obter $\mathrm{x} \in \mathbb{R}$ tal que $\mathrm{f}(\mathrm{x})=0$ estudamos alguns métodos clássicos na literatura, divididos em métodos intervalares e métodos abertos. Utilizaremos aqui o método de Newton-Raphson, que segundo [2], é dado por

$$
x_{n+1}=x_{n}-\frac{f\left(x_{n}\right)}{f^{\prime}\left(x_{n}\right)}
$$

para $\mathrm{n} \geq 1$, onde escolhemos o pontapé inicial $\mathrm{x}_{0}$.

Assim, para esse método escolhemos $\mathrm{x}_{0}$ e precisamos determinar uma tolerância tol $>0$ e um número máximo de iterações, uma vez que o método pode apresentar uma série de armadilhas. 
Em Matlab, escrevemos a função NR da seguinte forma:

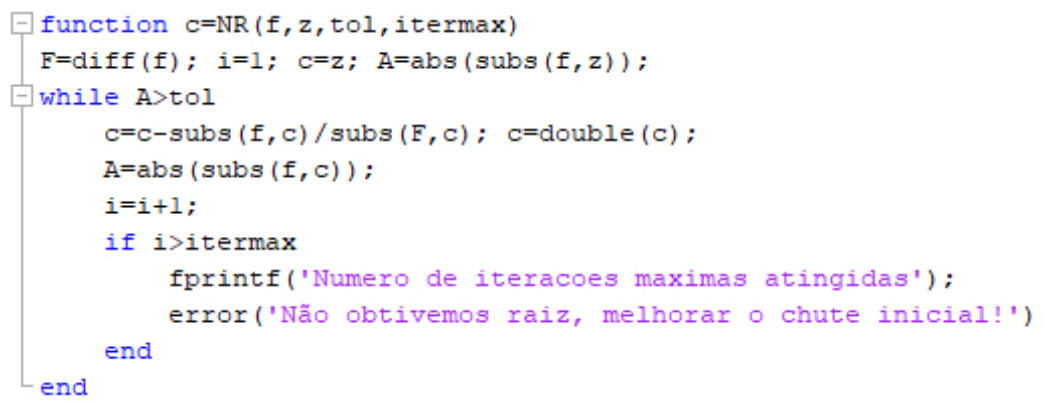

Ao algoritmo do sistema (3) acrescentamos as seguintes linhas:

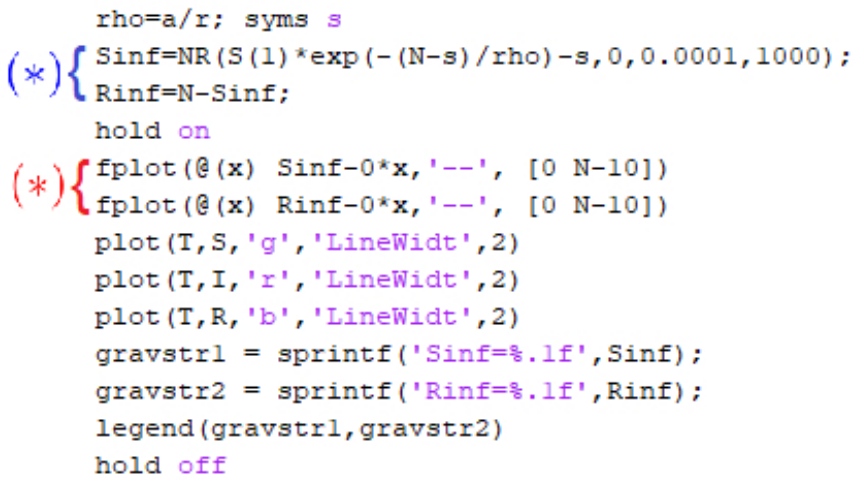

Detalhamos os seguintes pontos:

(*) Utilizamos o método de Newton-Raphson para obter os valores limite $\mathrm{S}(\infty)$ e $\mathrm{R}(\infty)$;

(*) Plotamos as funções constantes $\mathrm{y}_{1}=\mathrm{S}(\infty)$ e $\mathrm{y}_{2}=\mathrm{R}(\infty)$, que são as assíntotas para as soluções $\mathrm{S}(\mathrm{t})$ e $\mathrm{R}(\mathrm{t})$.

Na Figura 9 testamos o algoritmo para alguns valores de a e b considerando novamente $S(0)=1000$ e $\mathrm{I}(0)=10$.

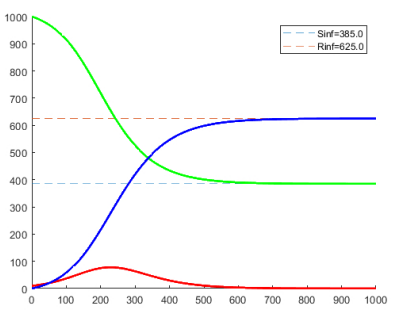

$\mathrm{a}=0.0275, \mathrm{r}=0.000042$.

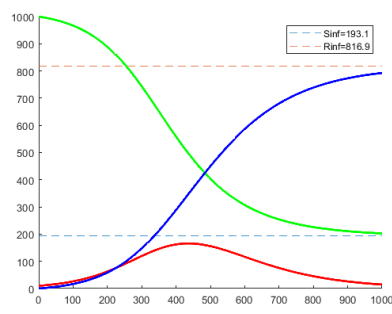

$\mathrm{a}=0.00986, \mathrm{r}=0.00001985$ 
Figura 9: Resultado do experimento para $\mathrm{S}(1)=1000 ; \mathrm{I}(1)=10 ; \mathrm{R}(1)=0$ e os parâmetros a e r como na imagem.

Como discutido, o resultado limite para uma epidemia está diretamente relacionado à taxa de remoção relativa $\rho$. Para uma determinada doença, a taxa de remoção relativa varia de acordo com a comunidade e, portanto, determina se uma epidemia pode ocorrer em uma comunidade e não em outra. $\mathrm{O}$ número de suscetíveis $\mathrm{S}_{0}$ obviamente também desempenha um papel importante. Por exemplo, se a densidade de suscetíveis for alta e a taxa de remoção de infectantes for baixa como por falta de assistência médica, isolamento inadequado ou até por ignorância - é provável que ocorra uma epidemia. Temos que $\mathrm{I}_{\max }$ fornece o número máximo de infectantes, enquanto $\mathrm{I}_{\text {total }}$ fornece o número total de pessoas infectadas em termos de $\rho=\mathrm{a} / \mathrm{r}, \mathrm{I}_{0}, \mathrm{~S}_{0}$ e N.

Essa discussão sugere que em casos graves, como a infecção da Covid-19, medidas de contenção devem ser tomadas, e um dos países que teve destaque nesse meio foi a Nova Zelândia. Até o fim da primeira semana de Maio de 2020, a Nova Zelândia havia registrado menos de 1500 casos confirmados de Covid-19 e 20 mortes. Em 23 de Março, um mês após o país ter registrado seu primeiro caso, a Nova Zelândia se comprometeu com uma estratégia de eliminação onde o Estado anunciou um bloqueio nacional estrito quando havia apenas 102 casos e nenhuma morte registrada. Sua rápida tomada de decisão ganhou elogios internacionais, inclusive da OMS. A abordagem realizada pelo país foi muito diferente do planejamento pandêmico usual, que historicamente se baseia em um modelo de mitigação e se concentra em atrasar a chegada do vírus, seguido de uma série de medidas para achatar a curva de contaminação. As restrições impostas envolveram o fechamento de escolas e locais de trabalho não essenciais, a proibição de reuniões sociais e severas restrições de viagem. Pôde-se constatar que o bloqueio total permitiu que o país colocasse em funcionamento os principais sistemas para gerenciar fronteiras de forma eficaz, além de rastrear contatos, testes e vigilância. Também, uma parcela muito grande da população foi testada e esses testes foram focados em pessoas com sintomas, com rastreamento de contatos próximos e contatos casuais. Com o sucesso da estratégia, a Nova Zelândia diminuiu suas restrições e sua economia foi se reabrindo lentamente, e evidentemente há discussões sobre como ela poderia abrir suas fronteiras e garantir que todos seus cidadãos estejam protegidos, principalmente as populações suscetíveis. Outro país que obteve sucesso no controle da epidemia foi a Austrália, mesmo que durante o percurso da epidemia não estivesse claro para o mundo qual foi ideia de eliminação.

\subsection{Aplicação dos métodos de Runge-Kutta}

Na maioria das epidemias, é difícil determinar quantos novos infecciosos existem por dia, uma vez que apenas os que são removidos, para assistência médica ou o que seja, podem ser contados. Os registros de saúde pública geralmente fornecem o número de infectantes por dia, semana ou mês. Portanto, para aplicar o modelo a situações epidêmicas reais, em geral, precisamos saber o número removido por unidade de tempo $\frac{\mathrm{dR}}{\mathrm{dt}}$ em função do tempo. Assim, utilizando a terceira equação de (3) e a equação (4), temos

$$
\frac{\mathrm{dR}}{\mathrm{dt}}=\mathrm{aI}=\mathrm{a}(\mathrm{N}-\mathrm{R}-\mathrm{S})
$$

Usando então a equação (10), obtemos o problema de valor inicial

$$
\left\{\begin{array}{l}
\frac{\mathrm{dR}}{\mathrm{dt}}=\mathrm{a}\left(\mathrm{N}-\mathrm{R}-\mathrm{S}_{0} \mathrm{e}^{-\frac{\mathrm{R}}{\rho}}\right), \\
\mathrm{R}(0)=0,
\end{array}\right.
$$


que só pode ser resolvido analiticamente de maneira implícita, e uma solução dessa forma não costuma ser conveniente. Obviamente, se conhecemos a, r, $\mathrm{S}_{0}$ e N, é simples calcular a solução numericamente. Utilizaremos o método de Runge-Kutta de quarta ordem a fim de entender melhor a dinâmica e os impactos desses dados e parâmetros. O clássico método de Runge-Kutta de quarta ordem, que é o de uso mais comum no dia a dia, é utilizado para obter a solução numérica Y do problema de valor inicial

$$
\mathrm{y}^{\prime}=\mathrm{f}(\mathrm{t}, \mathrm{y}), \quad \mathrm{y}\left(\mathrm{t}_{0}\right)=\mathrm{y}_{0} .
$$

O desenvolvimento do método é apresentado em [2] e procederemos de maneira semelhante ao que foi feito em [8] (P. 350). Assim, a fim de utilizar o método de Runge-Kutta para (14), em Matlab, escrevemos

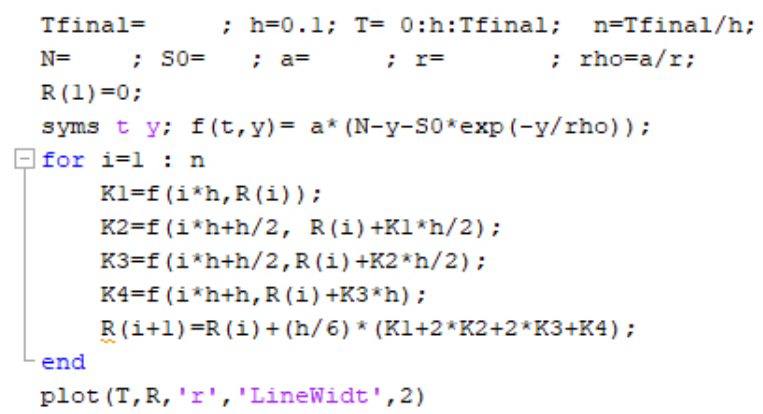

Exibimos então na Figura 10 os experimentos numéricos utilizando o método de Runge-Kutta de quarta ordem para Tfinal $=500, \mathrm{~h}=0.1$ e dados como lá indicado. Observamos que as escolhas de a e $\mathrm{r}$ influenciam diretamente no valor $\mathrm{R}(\infty)$, porém isso já era esperado devido à construção apresentada na seção anterior. Um detalhe que vale a pena discutir é sobre a geometria da curva e a diferença de comportamento no início dessa, que acontece apenas na última, porém, é comum ver em curvas de removíveis no dia a dia. Esse comportamento é diretamente ligado à pequenez do dado inicial de infectados, ou seja, tem relação com o quão próximo os suscetíveis iniciais $\mathrm{S}_{0}$ estão da população total N.

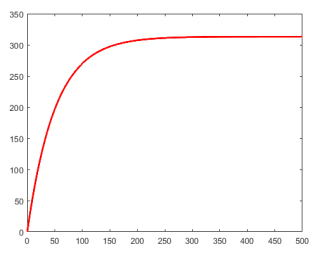

$\mathrm{S} 0=700$ e $\mathrm{r}=0.0000003$.

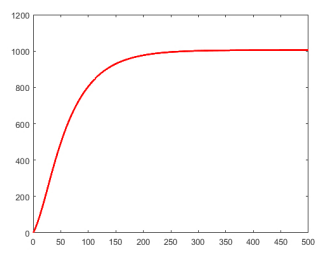

$\mathrm{S} 0=700$ e $\mathrm{r}=0.0001$.

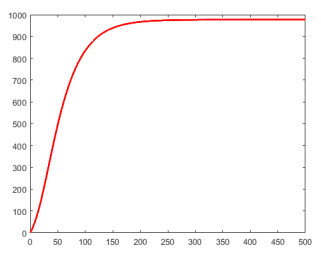

$\mathrm{S} 0=850$ e $\mathrm{r}=0.0001$.

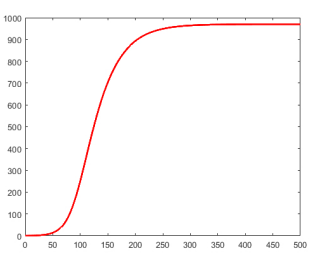

$\mathrm{S} 0=1009$ e $\mathrm{r}=0.0001$.

Figura 10: Resultado do experimento para (14) com dados $\mathrm{N}=1010$, a $=0.02$ e demais parâmetros como na imagem.

Convidamos o leitor que está aprendendo as técnicas apresentadas para testar sua curiosidade a fim de obter um ajuste como o apresentado na Figura 11. Nessas tentativas de ajuste podemos usar menos pontos na grade ou deixar o algoritmo de Runge-Kutta apresentado mais particular para o caso, evitando tantas substituições na função $\mathrm{f}(\mathrm{t}, \mathrm{y})$ que causam lentidão. Alternativa também é utilizar o método de Euler-explícito para ajustes iniciais (por sua velocidade) e então o método 
de Runge-Kutta (por sua precisão). É uma parte nada trivial obter os parâmetros que nos dão o ajuste ideal que procuramos; na próxima seção abordaremos esse problema em um caso particular fazendo uma hipótese sobre $\mathrm{R}_{0}$ e $\rho$.

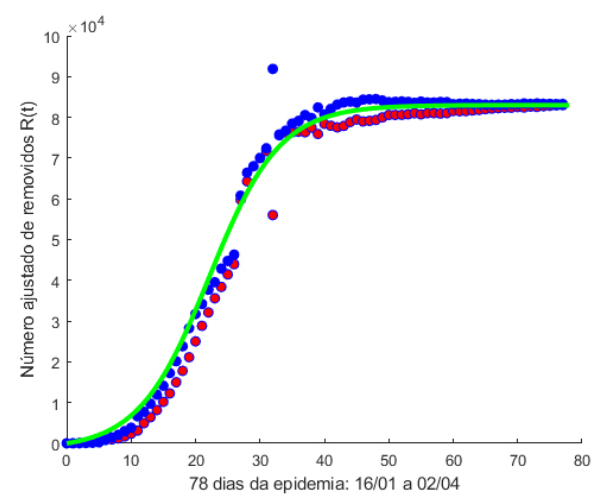

Figura 11: Ajuste da quantidade estimada de removidos na China.

\subsection{Aplicação da técnica de quadrados mínimos}

Normalmente, não conhecemos todos os parâmetros e, portanto, ao assumir que a epidemia é razoavelmente descrita pelo modelo estudado, precisamos executar um procedimento de melhor ajuste, como tentamos executar acima. Na prática, no entanto, geralmente ocorre que, se a epidemia não for grande, $\mathrm{R} / \rho$ é pequeno, ou pelo menos $\mathrm{R} / \rho<1$. A fim de contornar o último termo da equação em (14), consideremos a Série de Taylor de $\mathrm{e}^{-\mathrm{x}}$ em torno de 0:

$$
e^{-x}=1-x+\frac{x^{2}}{2}+r(x)
$$

onde $\mathrm{r}(\mathrm{x})=\mathrm{o}\left(\mathrm{x}^{3}\right)$. Então se $0<\mathrm{R} / \rho \ll 1$, uma boa aproximação para o último termo é dado por

$$
\mathrm{e}^{-\mathrm{R} / \rho} \approx 1-\frac{\mathrm{R}}{\rho}+\frac{\mathrm{R}^{2}}{2 \rho^{2}} .
$$

Assim, podemos então aproximar (14) por

$$
\left\{\begin{array}{l}
\frac{\mathrm{dR}}{\mathrm{dt}}=\mathrm{a}\left[\mathrm{N}-\mathrm{S}_{0}+\left(\frac{\mathrm{S}_{0}}{\rho}-1\right) \mathrm{R}-\frac{\mathrm{S}_{0} \mathrm{R}^{2}}{2 \rho^{2}}\right], \\
\mathrm{R}(0)=0 .
\end{array}\right.
$$

Notemos que a equação diferencial ordinária envolvida é separável, da forma

$$
\frac{\mathrm{dR}}{\mathrm{dt}}=\mathrm{a}\left(\mathrm{a}_{0}+\mathrm{b}_{0} \mathrm{R}+\mathrm{c}_{0} \mathrm{R}^{2}\right),
$$


donde podemos proceder de maneira simbólica utilizando integração trigonométrica para obter

$$
\left\{\begin{array}{l}
\mathrm{R}(\mathrm{t})=\frac{\mathrm{r}^{2}}{\mathrm{~S}_{0}}\left[\left(\frac{\mathrm{S}_{0}}{\rho}-1\right) \alpha \tanh \left(\frac{\alpha \mathrm{at}}{2}-\phi\right)\right] \\
\alpha=\left[\left(\frac{\mathrm{S}_{0}}{\rho}-1\right)^{2}+\frac{2 \mathrm{~S}_{0}\left(\mathrm{~N}-\mathrm{S}_{0}\right)}{\rho^{2}}\right]^{1 / 2} \\
\phi=\frac{\tanh ^{-1}\left(\frac{\mathrm{S}_{0}}{\rho}-1\right)}{\alpha} .
\end{array}\right.
$$

Para ver os detalhes da resolução dessa EDO separável citamos [5]. A taxa de remoção é então dada por

$$
\frac{\mathrm{dR}}{\mathrm{dt}}=\frac{\mathrm{a} \alpha^{2} \rho^{2}}{2 \mathrm{~S}_{0}} \operatorname{sech}^{2}\left(\frac{\alpha \mathrm{at}}{2}-\phi\right) .
$$

que envolve apenas 3 parâmetros, a $\alpha^{2} \rho^{2} / 2 \mathrm{~S}_{0}, \alpha$ a e $\phi$. Com epidemias que não são tão grandes, essa função do tempo oferece um bom ajuste para dados. Em contrapartida, em casos onde a doença é tal que sabemos o número real da classe removida, é R(t) em (16) que devemos utilizar. Se R/ $\rho$ não for pequeno, no entanto, devemos usar a equação diferencial (14) para determinar $\mathrm{R}(\mathrm{t})$. É possível ver alguns casos clássicos na literatura de bons ajustes de (17), como nos anos de 1905 e 1906 na epidemia de Praga de Bombaim, relatado em [6], ou na epidemia de gripe em um internato inglês em 1978, onde a revista médica britânica The Lancet apresentou um relatório com estatísticas detalhadas de uma epidemia de gripe em um internato, com um total de 763 meninos infectados.

Ajuste de curvas consiste em encontrar uma curva que se ajuste a uma série de pontos e que possivelmente cumpra uma série de parâmetros adicionais. Ajuste de curvas pode envolver tanto interpolação, onde é necessário um ajuste exato aos dados, quanto suavização, na qual é construída uma função "suave" que, aproximadamente, se ajusta aos dados. Citamos os métodos de polinômios interpoladores de Lagrange e Splines cúbicas para a interpolação exata e o método de quadrados mínimos para ajustes, ver [2].

A discussão feita anteriormente resumia que um ajuste para os dados dos removidos $\mathrm{R}$ pode ser dado via $f(t)=A \cdot \operatorname{sech}^{2}(p t+q), c o m$

$$
\left\{\frac{\mathrm{a} \alpha^{2} \rho^{2}}{2 \mathrm{~S}_{0}}=\mathrm{A} \quad \frac{\alpha \mathrm{a}}{2}=\mathrm{p} \quad-\phi=\mathrm{q} .\right.
$$

Então o problema a ser abordado nesse momento é obter os melhores parâmetros A, p e q, de modo que essas façam o melhor ajuste para dados

\begin{tabular}{|c|c|c|c|c|c|c|c|c|c|c|c|}
\hline $\mathrm{t}$ & 0 & 1 & 2 & 3 & 4 & 5 & 6 & 7 & 8 & $\cdots$ & $\mathrm{t}_{\mathrm{n}}$ \\
\hline $\mathrm{y}(\mathrm{t})$ & $\mathrm{y}_{0}$ & $\mathrm{y}_{1}$ & $\mathrm{y}_{2}$ & $\mathrm{y}_{3}$ & $\mathrm{y}_{4}$ & $\mathrm{y}_{5}$ & $\mathrm{y}_{6}$ & $\mathrm{y}_{7}$ & $\mathrm{y}_{8}$ & $\cdots$ & $\mathrm{y}_{\mathrm{n}}$ \\
\hline
\end{tabular}

Para isso, consideremos a função $\mathrm{E}: \mathbb{R}^{3} \rightarrow \mathbb{R}$ dada por

$$
E(A, p, q)=\sum_{i=1}^{n}\left[y_{i}-\operatorname{Asech}^{2}\left(p t_{i}+q\right)\right]^{2}
$$


Esse mínimo deve estar no ponto $(\mathrm{A}, \mathrm{p}, \mathrm{q})$ tal que $\nabla \mathrm{E}(\mathrm{A}, \mathrm{p}, \mathrm{q})=(0,0,0)$. O gradiente de $\mathrm{E}$ pode ser calculado via

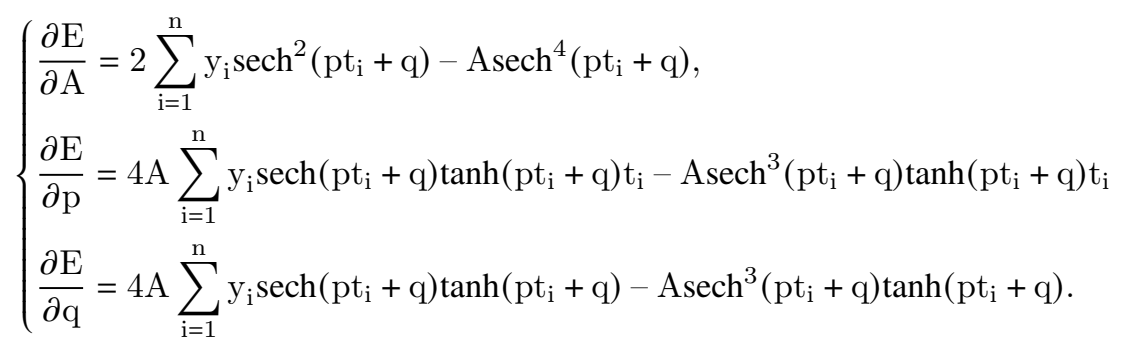

Assim, obter $(A, p, q)$ de modo que $\nabla \mathrm{E}(\mathrm{A}, \mathrm{p}, \mathrm{q})=(0,0,0)$ precisamos resolver o sistema não linear nas variáveis $A, p$ e $q$ dado por

$$
\left\{\begin{array}{l}
\sum_{i=1}^{n} y_{i} \operatorname{sech}^{2}\left(p t_{i}+q\right)-\operatorname{Asech}^{4}\left(p t_{i}+q\right)=0 \\
\sum_{i=1}^{n} y_{i} \operatorname{sech}\left(p t_{i}+q\right) \tanh \left(p t_{i}+q\right) t_{i}-\operatorname{Asech}^{3}\left(p t_{i}+q\right) \tanh \left(p t_{i}+q\right) t_{i}=0 \\
\sum_{i=1}^{n} y_{i} \operatorname{sech}\left(p t_{i}+q\right) \tanh \left(p t_{i}+q\right)-\operatorname{Asech}^{3}\left(p t_{i}+q\right) \tanh \left(p t_{i}+q\right) .=0
\end{array}\right.
$$

Resolver um sistema de equações não lineares é um problema evitado sempre que isso se faz possível. Utilizaremos o método de Newton, que é a generalização do método de NewtonRaphson para dimensão maior. Denotamos $\overrightarrow{\mathrm{F}}=\left(\mathrm{f}_{1}\left(\mathrm{x}_{1}, \ldots, \mathrm{x}_{\mathrm{n}}\right), \ldots, \mathrm{f}_{\mathrm{n}}\left(\mathrm{x}_{1}, \ldots, \mathrm{x}_{\mathrm{n}}\right)\right)$ e por $\mathrm{J}$ a matriz jacobiana de $\overrightarrow{\mathrm{F}}$. Segundo [2], o algoritmo do método de Newton para o sistema não linear $\overrightarrow{\mathrm{F}}(\overrightarrow{\mathrm{x}})=0$ é dado por

$$
\overrightarrow{\mathrm{x}}^{\mathrm{k}+1}=\overrightarrow{\mathrm{x}}^{\mathrm{k}}-\mathrm{J}\left(\overrightarrow{\mathrm{x}}^{\mathrm{k}}\right)^{-1} \overrightarrow{\mathrm{F}}\left(\overrightarrow{\mathrm{x}}^{\mathrm{k}}\right), \quad \mathrm{k} \geq 1
$$

onde estamos denotando $\overrightarrow{\mathrm{x}}^{\mathrm{k}}=\left(\mathrm{x}_{1}^{\mathrm{k}}, \cdots, \mathrm{x}_{\mathrm{n}}^{\mathrm{k}}\right)$. 
Então criamos a função Newton com as seguintes linhas em Matlab:

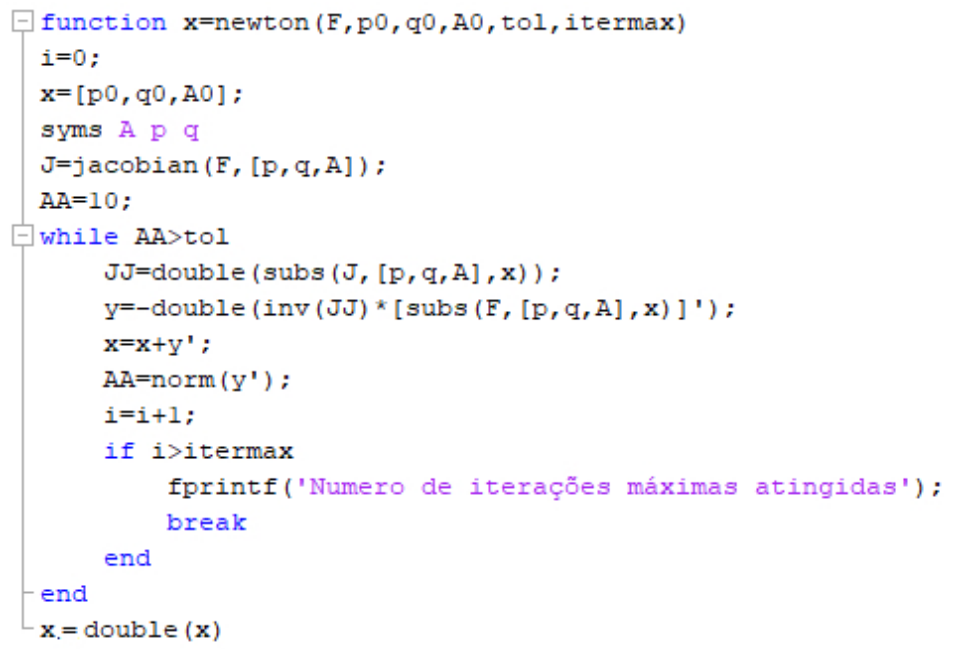

Apresentaremos um experimeto simbólico a fim de testar a teoria: iremos ajustar os dados dos vetores $\mathrm{T} \times$ Num_Mortes com a ideia acima, que é basicamente supor que quem se infectou foram apenas os que vieram a óbito. Por simplicidade, denotemos $\mathrm{M}=$ Num_Mortes. A fim de construir o campo F deste caso, tomemos as seguintes linhas em Matlab:

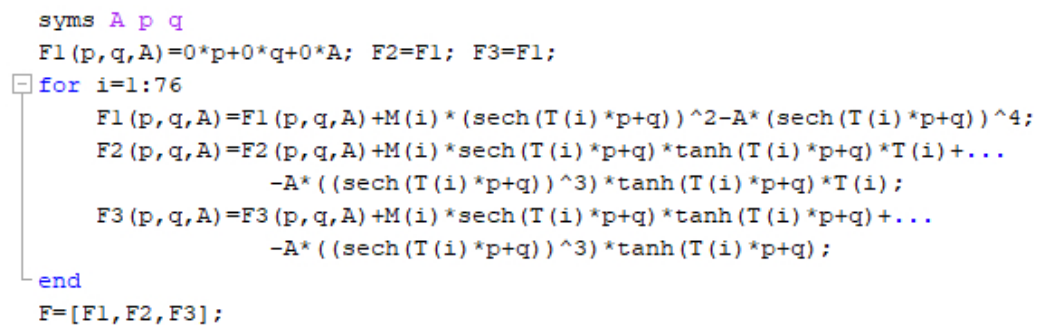

Assim, $\mathrm{X}=$ newton(F, 0.1,-3, 150, 0.0001, 10000) retorna

$$
\mathrm{X}=\left[\begin{array}{lll}
0.0759 & -2.2894 & 125.7793
\end{array}\right]
$$

Utilizamos esse resultado da seguinte forma:

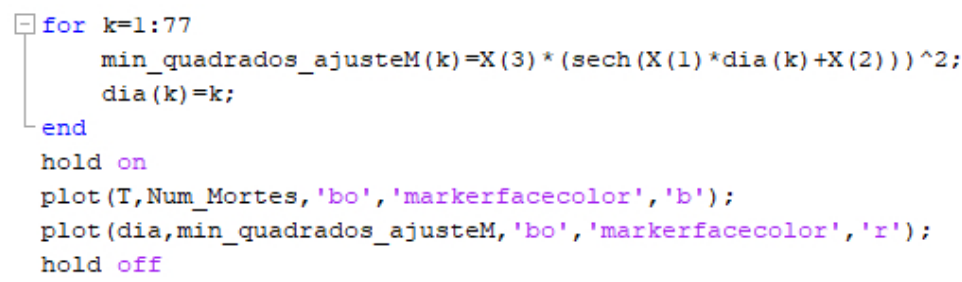

O resultado pode então ser visto à esquerda na Figura 12. Utilizando o que obtemos junto ao método de Runge-Kutta para (17), segue o ajuste feito à direita na Figura 12. 

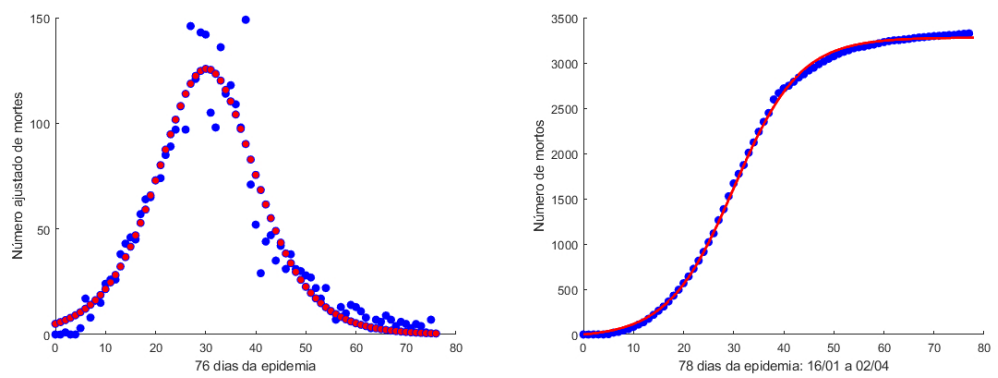

Figura 12: À esquerda o resultado via mínimos quadrados. À direita o ajuste da quantidade estimada de mortes na China pelo método de Runge-Kutta de quarta ordem.

Esses resultados seguem para mais exemplos de dados, onde sempre convém um bom pontapé inicial para que a função newton dê resposta. Muitas vezes é conveniente plotar $\operatorname{Asech}^{2}(p t+q)$ para alguns valores de A, p e q, onde de acordo com os dados que devem ser ajustados, podemos sugerir um pontapé inicial razoável. Ideias que foram usadas foi considerar para A um valor próximo ao dado mais alto, onde acima escolhemos $\mathrm{A}=150$, e para $\mathrm{p}$ e q podemos esperar que a relação $30 \mathrm{p}+\mathrm{q} \approx 0$ seja razoável, levando em consideração que a função $\mathrm{f}(\mathrm{t})=\operatorname{sech}^{2}(\mathrm{t})$ tem seu máximo nesse valor.

Os mesmos passos para a população de infectados seguem, isto é, para o ajuste de $\mathrm{T} \times \mathrm{Num} \_$Doentes por (17), considerando a adaptação do campo F com o vetor Num_Doentes em vez do vetor Num_Mortes e tomando $\mathrm{X}=$ newton $(\mathrm{F},-0.05,2,60000,0.0001,10000)$; obtemos

$$
\mathrm{X}=\left[-0.0656,2.3405,5.3706 \times 10^{4}\right]
$$

onde seguindo os mesmos passos obtemos o resultado que pode ser visto à esquerda na Figura 13. À direita da Figura 13 plotamos o ajuste para a população de infectados $\mathrm{I}(\mathrm{t})$ e as estimativas obtidas para $\mathrm{R}(\mathrm{t})$. Olhando à direita da Figura 13 e relembrando os primeiros experimentos para o modelo (3), é interessante como esse pode oferecer um bom ajuste ao problema.
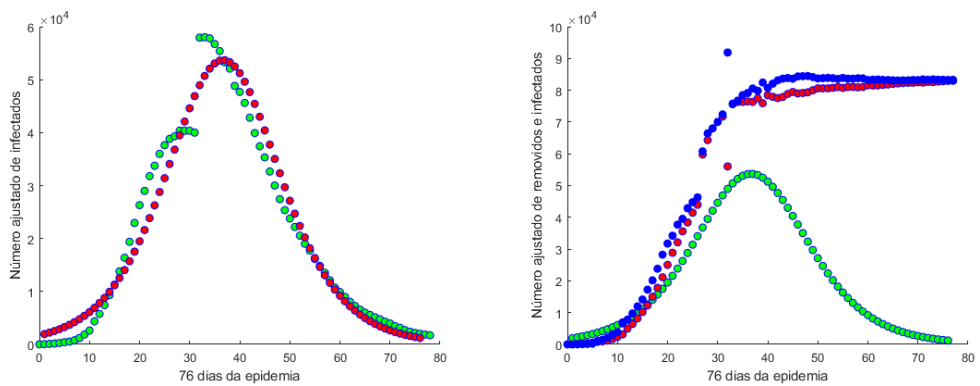

Figura 13: Ajuste da quantidade estimada de infectados na China.

\section{A. Plotando Listas em Matlab}


Apresentaremos de forma ultra-resumida os comandos de Listas do Matlab. As listas em Matlab serão utilizadas para obter soluções numéricas de alguns problemas. Em geral, elas são matrizes onde guardamos dados obtimos. Em Matlab, digitamos, por exemplo, as seguintes linhas:

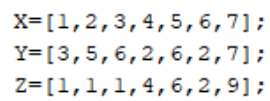

Temos três listas, que são matrizes $1 \times 7$. Temos, por exemplo, que X(1) devolve o primeiro elemento da lista, isto é, $\mathrm{X}(1)=1$. Outros exemplos são $\mathrm{Y}(4)=2, \mathrm{Z}(1)=1, \mathrm{Y}(6)=2$, e assim por diante. Comandos úteis que podemos usar são

$$
\begin{array}{ll}
4 & \mathrm{~A}=0: 10 ; \\
5 & \mathrm{~B}=0: 0.1: 1 ;
\end{array}
$$

que retornam

$$
\mathrm{A}=[1,2,3,4,5,6,7,8,9,10]
$$

e

$$
\mathrm{B}=[0,0.1,0.2,0.3,0.4,0.5,0.6,0.7,0.8,0.9,1.0] .
$$

Quando duas listas têm a mesma dimensão podemos usar o comando plot para plotar os seus pontos no plano cartesiano. Por exemplo, plot(X, Y) retorna os pontos $(1,3),(2,5),(3,6),(4,2)$, $(5,6),(6,2)$ e $(7,7)$ ligados por poligonais e de maneira similar para plot $(\mathrm{X}, \mathrm{Z})$, que em Matlab traz o seguinte resultado
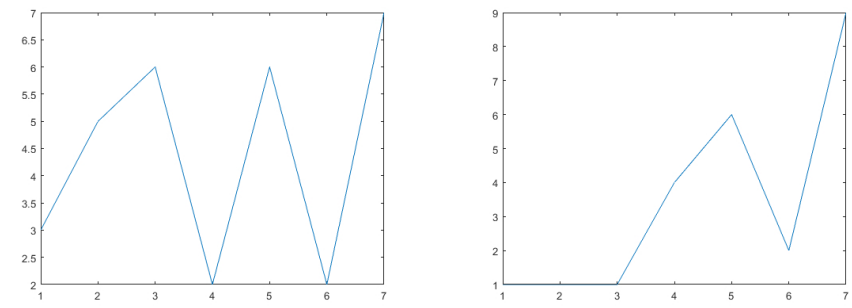

Podemos plotar alguns dados com pontos de alguma determinada cor, como por exemplo os comandos plot(X, Y,' bo',' markerfacecolor',', r') e plot(X, Z,' bo',' markerfacecolor',' b') retornam
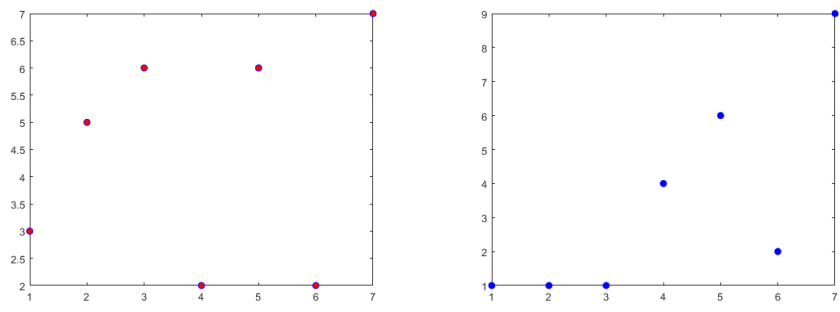

Observemos que ' $\mathrm{r}^{\prime}$ e ' $\mathrm{b}$ ' indicam as respectivas cores. Podemos usar outras formas de plotar, como por exemplo nas seguintes linhas

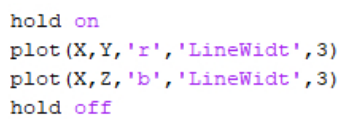


que colocam ambos os dados na mesma figura e tem o seguinte resultado

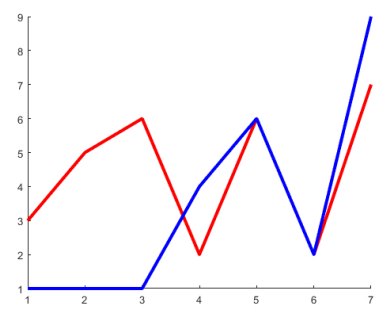

Por fim, um outro exemplo básico seguindo as linhas abaixo obtemos o resultado à esquerda:
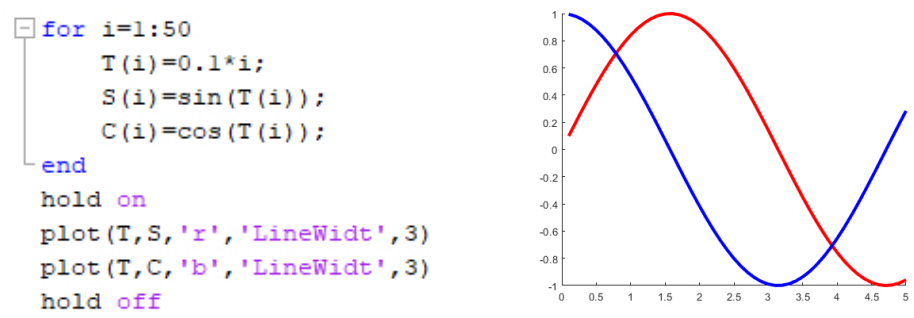

Esses são passos básicos que iremos utilizar ao longo de todo o texto. Para alternativas sugerimos uma busca em https://www.mathworks.com/help/matlab/ref/plot.html.

\section{B. Dados da epidemia do Covid-19 na China}

Dados da epidemia na China entre 16 de Janeiro a 02 de Abril com referência da legenda. Denotamos

- N.I. para número diário de novos infectados;

- N.M. para número diário de novas mortes;

- N.D. para número atual de doentes; 


\begin{tabular}{|c|c|c|}
\hline Dia & N.I. & N.M. \\
\hline \hline $16 / 01$ & 0 & 0 \\
\hline $17 / 01$ & 17 & 0 \\
\hline $18 / 01$ & 59 & 1 \\
\hline $19 / 01$ & 77 & 0 \\
\hline $20 / 01$ & 77 & 0 \\
\hline $21 / 01$ & 149 & 3 \\
\hline $22 / 01$ & 571 & 17 \\
\hline $23 / 01$ & 259 & 8 \\
\hline $24 / 01$ & 444 & 16 \\
\hline $25 / 01$ & 688 & 15 \\
\hline $26 / 01$ & 769 & 24 \\
\hline $27 / 01$ & 1771 & 26 \\
\hline $28 / 01$ & 1459 & 26 \\
\hline $29 / 01$ & 1737 & 38 \\
\hline $30 / 01$ & 1981 & 43 \\
\hline $31 / 01$ & 2099 & 46 \\
\hline $01 / 02$ & 2589 & 45 \\
\hline $02 / 02$ & 2825 & 57 \\
\hline $03 / 02$ & 3233 & 64 \\
\hline $04 / 02$ & 3886 & 65 \\
\hline $05 / 02$ & 3694 & 73 \\
\hline $06 / 02$ & 3143 & 74 \\
\hline $07 / 02$ & 3385 & 85 \\
\hline $08 / 02$ & 2656 & 89 \\
\hline $09 / 02$ & 3062 & 97 \\
\hline $10 / 02$ & 2478 & 108 \\
\hline
\end{tabular}

\begin{tabular}{|c|c|c|}
\hline Dia & N.I. & N.M. \\
\hline \hline $11 / 02$ & 2015 & 97 \\
\hline $12 / 02$ & 15152 & 146 \\
\hline $13 / 02$ & 5090 & 121 \\
\hline $14 / 02$ & 2641 & 143 \\
\hline $15 / 02$ & 2009 & 142 \\
\hline $16 / 02$ & 2048 & 105 \\
\hline $17 / 02$ & 1886 & 98 \\
\hline $18 / 02$ & 1749 & 136 \\
\hline $19 / 02$ & 817 & 114 \\
\hline $20 / 02$ & 889 & 118 \\
\hline $21 / 02$ & 397 & 109 \\
\hline $22 / 02$ & 648 & 98 \\
\hline $23 / 02$ & 214 & 149 \\
\hline $24 / 02$ & 508 & 71 \\
\hline $25 / 02$ & 406 & 52 \\
\hline $26 / 02$ & 433 & 29 \\
\hline $27 / 02$ & 327 & 44 \\
\hline $28 / 02$ & 427 & 47 \\
\hline $29 / 02$ & 573 & 35 \\
\hline $01 / 03$ & 202 & 42 \\
\hline $02 / 03$ & 125 & 31 \\
\hline $03 / 03$ & 119 & 38 \\
\hline $04 / 03$ & 139 & 31 \\
\hline $05 / 03$ & 143 & 30 \\
\hline $06 / 03$ & 99 & 28 \\
\hline $07 / 03$ & 44 & 27 \\
\hline
\end{tabular}

\begin{tabular}{|c|c|c|}
\hline Dia & N.I. & N.M. \\
\hline $08 / 03$ & 40 & 22 \\
\hline $09 / 03$ & 19 & 17 \\
\hline $10 / 03$ & 24 & 22 \\
\hline $11 / 03$ & 15 & 11 \\
\hline $12 / 03$ & 20 & 7 \\
\hline $13 / 03$ & 11 & 13 \\
\hline $14 / 03$ & 20 & 10 \\
\hline $15 / 03$ & 16 & 14 \\
\hline $16 / 03$ & 21 & 13 \\
\hline $17 / 03$ & 13 & 11 \\
\hline $18 / 03$ & 34 & 8 \\
\hline $19 / 03$ & 39 & 3 \\
\hline $20 / 03$ & 41 & 7 \\
\hline $21 / 03$ & 46 & 6 \\
\hline $22 / 03$ & 39 & 9 \\
\hline $23 / 03$ & 78 & 7 \\
\hline $24 / 03$ & 47 & 4 \\
\hline $25 / 03$ & 67 & 6 \\
\hline $26 / 03$ & 55 & 5 \\
\hline $27 / 03$ & 54 & 3 \\
\hline $28 / 03$ & 45 & 5 \\
\hline $29 / 03$ & 31 & 4 \\
\hline $30 / 03$ & 48 & 1 \\
\hline $31 / 03$ & 36 & 7 \\
\hline $01 / 04$ & 35 & 6 \\
\hline $02 / 04$ & 31 & 4 \\
\hline
\end{tabular}

Tabela 1: Dados da epidemia na China entre 16 de Janeiro a 02 de Abril referentes a número diários de mortes e pessoas infectadas. Esses dados podem ser obtidos em https://www.worldometers.info/ ou no site da Universidade Johns Hopkins em https://coronavirus.jhu.edu/, por exemplo. 


\begin{tabular}{|c|c|c|c|c|c|c|c|}
\hline Dia & N.D. & Dia & N.D. & Dia & N.D. & Dia & N.D. \\
\hline $16 / 01$ & 24 & $05 / 02$ & 26302 & $25 / 02$ & 47672 & $16 / 03$ & 9898 \\
\hline $17 / 01$ & 41 & $06 / 02$ & 28984 & $26 / 02$ & 45604 & $17 / 03$ & 8976 \\
\hline $18 / 01$ & 94 & $07 / 02$ & 31774 & $27 / 02$ & 42808 & $18 / 03$ & 8056 \\
\hline $19 / 01$ & 170 & $08 / 02$ & 33738 & $28 / 02$ & 39919 & $19 / 03$ & 7263 \\
\hline $20 / 01$ & 260 & $09 / 02$ & 35982 & $29 / 02$ & 37414 & $20 / 03$ & 6569 \\
\hline $21 / 01$ & 406 & $10 / 02$ & 37626 & $01 / 03$ & 35329 & $21 / 03$ & 6013 \\
\hline $22 / 01$ & 529 & $11 / 02$ & 38800 & $02 / 03$ & 32652 & $22 / 03$ & 5549 \\
\hline $23 / 01$ & 771 & $12 / 02$ & 39302 & $03 / 03$ & 30004 & $23 / 03$ & 5120 \\
\hline $24 / 01$ & 1208 & $13 / 02$ & 40364 & $04 / 03$ & 27433 & $24 / 03$ & 4735 \\
\hline $25 / 01$ & 1870 & $14 / 02$ & 40364 & $05 / 03$ & 25352 & $25 / 03$ & 4291 \\
\hline $26 / 01$ & 2613 & $15 / 02$ & 40351 & $06 / 03$ & 23784 & $26 / 03$ & 3947 \\
\hline $27 / 01$ & 4349 & $16 / 02$ & 40007 & $07 / 03$ & 22177 & $27 / 03$ & 3460 \\
\hline $28 / 01$ & 5739 & $17 / 02$ & 57934 & $08 / 03$ & 20533 & $28 / 03$ & 3128 \\
\hline $29 / 01$ & 7417 & $18 / 02$ & 58023 & $09 / 03$ & 19016 & $29 / 03$ & 2691 \\
\hline $30 / 01$ & 9308 & $19 / 02$ & 57805 & $10 / 03$ & 17721 & $30 / 03$ & 2396 \\
\hline $31 / 01$ & 11289 & $20 / 02$ & 56729 & $11 / 03$ & 16145 & $31 / 03$ & 2161 \\
\hline $01 / 02$ & 13784 & $21 / 02$ & 55391 & $12 / 03$ & 14831 & $01 / 04$ & 2004 \\
\hline $02 / 02$ & 16369 & $22 / 02$ & 53284 & $13 / 03$ & 13526 & $02 / 04$ & 1864 \\
\hline $03 / 02$ & 19381 & $23 / 02$ & 52092 & $14 / 03$ & 12094 & $03 / 04$ & 1727 \\
\hline $04 / 02$ & 22942 & $24 / 02$ & 48824 & $15 / 03$ & 10734 & $04 / 04$ & 1562 \\
\hline
\end{tabular}

\section{Referências}

[1] W.E. Boyce, R.C DiPrima, Equações diferenciais elementares e problemas de valores de contorno. $9^{\mathrm{a}}$ ed, LTC.

[2] L.R. Burden, J.D. Faires. Análise Numérica, $8^{a}$ edição. [S.l.]: São Paulo, 2003.

[3] V. Capasso, Mathematical Structures of Epidemic Systems, Lecture Notes in Biomath. 97, Springer Verlag (1993).

[4] O. Diekmann, J.A.P. Heesterbeek, Mathematical Epidemiology of Infectious Diseases Wiley series in Mathematical and Computational Biology, Wiley (2000).

[5] S.H. de Jesus Nicola, A matemática e a epidemia, Professor de Matemática Online (PMO), 2020.

[6] J.D. Murray, Mathematical Biology I - An Introduction. Third Edition, Springer, 2000.

[7] M. Iannelli, The Mathematical Modeling of Epidemics. Mathematical models in Life Science: Theory and Simulation, University of Trento, 2005.

[8] B. Telch, C. Cominato, R. Cardoso, Desenvolvimento da disciplina de Cálculo Numérico tendo um modelo em Ecologia como eixo norteador. Professor de Matemática Online (PMO), 2020. 
Júlia Beatriz Meira

Universidade do Estado de Santa Catarina

$<$ juliameira12@gmail.com>

Bruno Telch dos Santos

Universidade do Estado de Santa Catarina

$<$ telchbruno@gmail.com>

Recebido: $18 / 07 / 2020$

Publicado: $13 / 04 / 2021$ 